\title{
ABSTRACTS
}

\section{CGS 41st Annual Scientific Meeting: Book of Abstracts}

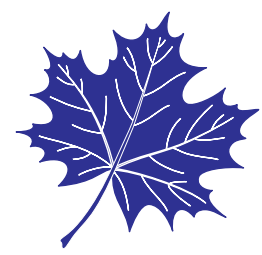

\section{FINALISTS FOR THE WILLARD AND PHOEBE THOMPSON AWARD}

\section{Evaluation of a Student-Older Adult Telephone \\ Befriending Program to Reduce Social Isolation during the COVID-19 Pandemic: A Pilot Evaluation}

Manan Ahuja, Kaitlin Lewis, Leanne Kim, Sawayra Owais, Jordana Compagnone, Laura Fallico, Francine Fishbein, Isabella Stefanova, Kai Man Xu, Mary Boulos, Shannon Gui

Michael G. DeGroote School of Medicine, McMaster University

Background: Social isolation and loneliness are associated with many adverse health outcomes. The COVID-19 pandemic has increased this risk and disproportionately affected older adults, particularly those living alone with frailty and limited supports. The McMaster Phone-a-Friend Program (PFP) was established, from the UofT SSIPP initiative, with the goal of reducing social isolation through telephone befriending. This study's goal is to evaluate the effectiveness of the program in reducing social isolation and explore its long-term sustainability after the pandemic.

Methods: Community-dwelling older adults at risk for social isolation during the pandemic in the Hamilton, Waterloo and Niagara regions of Canada were identified by primary care providers and matched to trained student volunteers, providing social interaction through weekly telephone calls. Older adults completing $\geq 4$ calls were solicited to complete an 8-item self-report telephone questionnaire to determine the program's impact with responses ranging from "Strongly Agree" to "Strongly Disagree".

Results: To date, the PFP has matched 64 older adults and student volunteers. Twenty-seven older adults (mean age 74 years; $73 \%$ female; $74 \%$ living alone) participated in the survey. The majority of participants agreed or strongly agreed the program made them feel less lonely (78\%), improved their quality of life $(70 \%)$ and that they would continue participating in the program after the pandemic (74\%). Qualitative feedback suggests potential areas for program enhancement include options for self-referrals, call schedule flexibility and interactive means of communication, such as videoconferencing.

Discussion: Student-led telephone befriending programs appear to be an effective, sustainable and generalizable method of reducing patient-reported social isolation and loneliness, after at least one month of participation, among communitydwelling older adults during the pandemic.

Conclusions: Telephone-befriending programs are a feasible and safe intervention to combat social isolation during the pandemic.

\section{Updated Inventory and Projected Requirements for Specialist Physicians in Geriatrics}

Monisha Basu ${ }^{1}$, Tracy Cooper ${ }^{2}$, Kelly Kay ${ }^{3}$, David B. Hogan ${ }^{4}$, José A. Morais ${ }^{5}$, Frank Molnar ${ }^{6}$, Robert E. Lam ${ }^{7}$, Michael J. Borrie ${ }^{8}$

${ }^{1}$ University of Toronto Medicine, ${ }^{2}$ St. Joseph's Health Care, London, ${ }^{3}$ Provincial Geriatrics Leadership Office, ${ }^{4}$ Division of Geriatric Medicine, Cumming School of Medicine, University of Calgary, ${ }^{5}$ Division of Geriatrics and Research Institute of the McGill University Health Centre, McGill University, ${ }^{6}$ Eastern Ontario Regional Geriatric Program. University of Ottawa and Ottawa Hospital Department of Medicine, ${ }^{7}$ Home Based Primary Care Program, Unison Health and Community Services, ${ }^{8}$ Division of Geriatric Medicine, Schulich School of Medicine \& Dentistry, Western University

Background: Geriatric physician resource planning for the predicted growth of Canadians aged 65+ and demand for specialized health services is needed. We updated the 2011 Canadian Geriatrics Society physician resource inventory and created projections for 2025 and 2030.

Methods: The number and full-time equivalents (FTEs) of geriatricians and Care of the Elderly (COE) physicians working in Specialized Geriatric Services (SGS) were determined. The 2025 and 2030 geriatric physician complement were estimated by accounting for anticipated retirements and new trainees. To determine current and future geriatrician requirements the Ontario 1991 Delphi consensus ratio of 1.25/10,000 population $65+$ was used. 
Results: Between 2011 and 2019 the number of geriatricians and COE physicians increased from 276 (235.8 FTEs) and 128 (89.9 FTEs) respectively to 376 (319.6 FTEs) and 30 percent 354 (115.5 FTEs working in SGS). The increase in geriatricians and COE physicians working in SGS did not keep pace with the $65+$ population growth. The geriatrician numbers increased primarily in Ontario and British Columbia and decreased in Nova Scotia and Manitoba. The absolute shortfall will continue to grow for 2025 and 2030, when it will be 552 in 2025 and 587 in 2030.

Discussion: The supply gap of geriatricians and COE physicians is projected to widen in 2025 and 2030. Comparison to the number of pediatricians practicing in Canada by the Canadian Medical Association (2019) shows a disparity in the specialist physician human resources available to the population $65+$ with 0.8 geriatricians per 100,000 population, compared to the population $0-19$ with 7.8 pediatricians per 100,000 population.

Conclusions: Increased recruitment to the field, greater use of interdisciplinary team-based care, enhanced funding models and more fully involving COE physicians in SGS would help reduce this gap.

\section{Evaluating the Effectiveness of the Reverse Stethoscope Technique for Hearing-Impaired Patients: A Speech- Intelligibility-Index Comparison}

Raphaelle Koerber ${ }^{1}$, Linh Vaccarello ${ }^{2}$, Allan $\mathrm{Ho}^{2}$

${ }^{1}$ McMaster School of Medicine, ${ }^{2}$ University of Alberta

Faculty of Medicine and Dentistry

Background: The reverse stethoscope technique is a frequently cited 'pearl' for communicating with patients with hearing loss. Here, a clinician places their stethoscope earpieces into the ears of a patient with hearing loss and speaks into the chest piece. As this technique has never been studied academically, we evaluated the effects of this technique on Speech Intelligibility Index scores for hearing-impaired adults, as compared to the effects of natural speech and a Pocketalker ${ }^{\circledR}$ voice amplifier.

Methods: The International Speech Test Signal was presented to four Littman ${ }^{\circledR}$ stethoscope models and a Pocketalker ${ }^{\circledR}$ personal voice amplifier using an Audioscan ${ }^{\circledR}$ test box for acoustic devices. The outputs of the stethoscopes and voice amplifier were measured by the test box microphone and coupler across the frequency spectrum of speech. The Speech Intelligibility Index scores of the resulting speech were calculated for each device in relation to ten common hearing losses representing the population of hearing-impaired elderly patients. These scores were compared to the Speech Intelligibility Index scores for natural speech.

Results: For each of the common hearing losses, the speech signal emitted by the stethoscopes yielded lower Speech Intelligibility Index scores as compared to natural speech. In contrast, the voice amplifier improved speech intelligibility.

Discussion: We reaffirm the utility of the stethoscope in transmitting the low frequency sounds for which it is designed. However, speech intelligibility depends on higher frequency sounds and stethoscopes attenuate these frequencies. As a result, the reverse stethoscope technique decreases, rather than improves, Speech Intelligibility Index scores.

Conclusions: For patients with hearing loss, the reverse stethoscope technique degrades the volume and clarity of speech. Instead, health care providers should rely on clearly articulated natural speech and, whenever possible, personal voice amplifiers.

\section{Fear of Falling Among Older Adults: A Scoping Review of Recent Literature}

Scott MacKay ${ }^{1}$, Patricia Ebert ${ }^{2}$, Cathy Harbridge ${ }^{3}$, David Hogan ${ }^{4}$

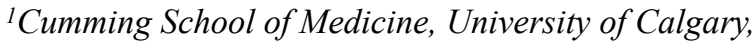

${ }^{2}$ Department of Psychology \& the Hotchkiss Brain Institute, University of Calgary; Seniors Health, Specialized

Geriatric Services, Alberta Health Services, ${ }^{3}$ Calgary Fall

Prevention Clinic, Specialized Geriatric Services, Calgary

Regional Health Authority, ${ }^{4}$ Division of Geriatric Medicine, Department of Medicine, Cumming School of Medicine, University of Calgary

Background: Fear of falling (FOF) is prevalent among older adults and associated with adverse health outcomes. Over recent years a substantial body of research has emerged on its epidemiology, associated factors, and consequences. We performed a scoping review to summarize the FOF literature published between April 2015 and March 2020 to inform current practice and identify gaps in the literature that could be addressed in future research.

Methods: A search of the PubMed database using the term "fear of falling" restricted to English publications appearing between April 1, 2015 and March 31, 2020 that included persons $65+$ was conducted. A total of 439 articles were identified, 56 selected for full-text review, and 46 retained for data extraction and synthesis.

Results: The majority of included studies had a cross-sectional design. The factors most consistently associated with FOF were older age, female gender, previous falls, worse physical performance, and depressive symptoms. Studies that measured FOF with a single question reported a significantly lower prevalence of FOF than those using the Falls Efficacy Scale, a continuous measure that assesses concern about falling during social and physical activities. FOF was associated with higher likelihoods of future falls, short-term mortality, and functional decline.

Discussion: Comparisons between studies were limited by the inconsistent definition and measurement of FOF, falls, and other characteristics. Consensus on how to measure FOF 
and which participant characteristics and outcomes to evaluate would address this issue. Gaps in the available literature include the relationship between FOF and cognitive, psychological, social, and environmental factors.

Conclusions: This scoping review provides a comprehensive overview of recent FOF literature that could be used to inform current practice and future research for this concern.

\section{A Multi-Specialist Telemedicine-Based Consultation Program for Homebound Older Adults}

Alfiya Mukharyamova, Nicoda Foster, Irene Wang, Pauline P. Kabitsis, Mary Ann Hamelin, Nga Troung, Janny Lee,

Samir K. Sinha

Sinai Health System

Background: A Multi-Specialist Telemedicine (TM) Consultation Program was established to improve access to specialist care and enhance the continuity of care for homebound older adults in Toronto, Canada. This study aimed to describe the program's implementation, the characteristics of its enrolled patients and patient/caregiver and provider satisfaction with the program in its first 5 years.

Methods: A prospective observational study was conducted during the first 5 years of the program's operation (2015-2020). Descriptive statistics were generated that included the number of unique patients seen, their demographics and the types of consultations being conducted. We also analyzed satisfaction surveys from 59 patients and caregivers and 22 providers.

Results: The average age of the 216 unique homebound patients served was 84 years, with an average age adjusted Charlson Comorbidity Index (CCI) of 6.55. These patients received 1003 consultations and follow-up visits from 21 different specialties. $86 \%$ of providers reported that the program enhanced their delivery of patient care and $100 \%$ of surveyed patients and caregivers reported being very satisfied with the program.

Discussion: This study describes the implementation of a TM program aimed to provide older homebound patients with improved access to specialist care. The fact that the program's operations continued unaffected throughout the COVID-19 pandemic further supports the fact that TM programs can be very effective in ensuring continuity of care, especially when people may be motivated to postpone seeking in-person medical care.

Conclusions: This program was successful in maintaining continuity of care for older homebound adults while maintaining high satisfaction rates among providers, patients and caregivers. With the field of TM receiving renewed attention during the COVID-19 pandemic, this program may help to provide guidance for others considering instituting similar models of care.

\section{Canadian Pharmacy Residency Program Barriers to the Inclusion of Geriatric Rotations}

Cody Thompson, Morgan Bharadia, Cheryl Sadowski University of Alberta

Background: The integration of geriatrics in pharmacy residency programs in Canada has not been studied. The purpose of our research was to describe the approach residency coordinators (RC) took for geriatrics in their programs. The focus of this presentation is on the barriers and plans identified by the $\mathrm{RC}$ for including geriatrics.

Methods: RCs from residency programs across Canada were surveyed through a semi-structured interview regarding the geriatric opportunities offered within their programs. The interview questions included details about the requirements for rotations, preceptors, resources, and future planning in regards to geriatrics.

Results: A total of $42 \mathrm{RC}$ from 44 programs were contacted. Of the 36 programs that participated, 29 were PGY1, with only 2 providing mandatory geriatrics rotations. Of the 10 programs that failed to offer any geriatrics rotations 7 indicated they did not have the capacity to support a rotation, 5 because they did not have an adequate patient population, and 3 because of insufficient preceptors. Nine of the 10 programs had no plans to address the lack of geriatrics. Of the 7 PGY2 rotations 5 did not offer a geriatrics rotation and 2 indicated they did not have the capacity. None of the 5 had plans to address geriatrics.

Discussion: The lack of geriatrics rotations in pharmacy residency programs is consistent with research from the US. This fails to address the preparation of pharmacists for hospital practice, or for pharmacy leadership positions within the healthcare system where resident graduates are often directed.

Conclusions: Pharmacy residency programs in Canada lack geriatrics training, primarily attributed to the patient population in the program setting and the lack of adequately prepared preceptors. There is insufficient planning to address this gap in pharmacy education.

\section{ELIGIBLE FOR THE RÉJEAN HÉBERT CANADIAN INSTITUTES OF HEALTH RESEARCH - INSTITUTE ON AGING PRIZE}

\author{
Assessing the Accuracy of International \\ Classification of Diseases (ICD) Coding for Delirium \\ Victoria Chuen ${ }^{1}$, Adrian Chan ${ }^{2}$, Shabbir Alibhai ${ }^{3}$, \\ Vicky Chau ${ }^{3}$ \\ ${ }^{1}$ McMaster University, ${ }^{2}$ University of Saskatchewan, \\ ${ }^{3}$ University Health Network
}


Background: Delirium is common amongst hospitalized older adults and is associated with increased morbidity, admission duration, and costs. Following discharge, charts are coded according to the World Health Organization International Classification of Diseases 10th edition (ICD-10) standards. The accuracy and completeness of these codes have implications for reimbursement, research and service programming. We assessed the accuracy of ICD coding for delirium and its relationship with delirium documentation in discharge summaries.

Methods: We performed a multi-centre retrospective chart review to identify hospitalized patients aged $\geq 65$ years with delirium using the Chart-based Delirium Identification Instrument, a validated tool for identifying delirium in the chart. Administrative discharge data were used to determine the presence of an ICD-10 code for delirium (F05). Charts of delirious patients were further divided based on whether "delirium" was documented in the discharge summary and compared for the presence of a F05 code.

Results: Among patients identified to have delirium through chart review $(\mathrm{n}=108), 46 \%$ of charts $(\mathrm{n}=50)$ received a F05 code. 755 charts not found to have delirium served as controls. The F05 code had a sensitivity of $46.3 \%$ and specificity of $99.6 \%$ for delirium, with a positive predictive value of $94.3 \%$ and a negative predictive value of $92.8 \% .67 \%$ of charts with "delirium" documented in the discharge summaries appropriately received a F05 code.

Conversely, when "delirium" was not documented, $12 \%$ of charts received a F05 code.

Discussion: While the ICD-10 administrative code for delirium is highly specific, it cannot reliably identify delirium given its poor sensitivity. Documentation of "delirium" in the discharge summary may contribute to improved coding accuracy.

Conclusions: ICD-10 administrative data inadequately captures delirium incidence. Further work is required to improve the accuracy of ICD coding for delirium.

\section{Emotional Impact On Health Care Providers Participating in Medical Assisted in Dying (Maid): A Systematic Review And Qualitative Meta-Synthesis}

Saumil Dholakia ${ }^{1}$, Alireza Bagheri ${ }^{2}$, Alexander (Sandy)

Simpson ${ }^{2}$

${ }^{I}$ GERIMEDRISK, McMaster University, ${ }^{2}$ CAMH

Background: Medical Assistance in Dying (MAiD) traverses challenging and emotionally overwhelming territories: Health Care Providers (HCP) across jurisdictions bridge the divide between normative yet opposing values of sanctity of life vs. dignity in death and dying and client's autonomous wish to die vs. provider's best interests, resulting into a myriad of affective responses.
Methods: A systematic search of 4 databases (OVID Medline, CINAHL, EMBASE, and Scopus) and grey literature. Key author, citation, and reference searches were also undertaken. Papers were included if they presented qualitative data regarding the emotional impact on HCPs involved in MAiD. Studies were restricted to english language. Analysis was conducted using meta-synthesis.

Results: Search identified 4522 papers. After applying inclusion criteria and The Joanna Briggs Institute Critical appraisal tool for Qualitative research, 34 papers were included in the meta-synthesis. Three distinct emotional themes were identified-(1) Strong, internalized emotions including moral distress, (2) actionable, externalized role-based emotions based on personal/moral/professional values, and (3) Reflective emotions that point towards MAiD being a 'sense making process'.

Discussion: The results show that HCPs are more reflective of the social discourse around MAiD in jurisdictions that legislate MAiD with emphasis on alleviating intolerable suffering. In jurisdictions that legislate MAiD as a means to alleviate intolerable suffering of terminally ill medical conditions, HCPs experience emotions based on their individual personal/moral and professional values.

Conclusions: This systematic review and meta-synthesis maps the current discourse amongst health care providers - it attempts to answer the question of "what it means at an emotional level' for a health care provider to consider/engage in MAiD. The presentation discusses the role of legal frameworks and the position of the health care provider in shaping the emotional discourse around MAiD.

\section{The Frail-Fit 30 Study: Factors Influencing 30-day Mortality in Frail patients Admitted to ICU}

David Hewitt ${ }^{1}$, Michael Ratcliffe ${ }^{2}$, Dr Malcolm Booth ${ }^{3}$ ${ }^{I} \mathrm{NHS},{ }^{2}$ University of Glasgow, ${ }^{3}$ University of Glasgow, $N H S G G \& C$

Background: Frailty is a multi-dimensional syndrome of reduced reserve, resulting from overlapping physiological decrements across multiple systems. The contributing factors, temporality and magnitude of frailty's effect on mortality after ICU admission are unclear. This study assessed frailty's impact on mortality and life sustaining therapy (LST) use, following ICU admission.

Methods: This single-centre retrospective observational cohort study analysed data collected prospectively in Glasgow Royal Infirmary ICU. Of 684 eligible patients, 171 were frail and 513 were non-frail. Frailty was quantified using the Rockwood Clinical Frailty Scale (CFS). All patients were followed up 1-year after ICU admission. The primary outcome was all-cause mortality at 30-days post-ICU admission. Key secondary outcomes included mortality at 1- year and LST use. 
Results: Frail patients were significantly less likely to survive 30 -days post-ICU admission $(61.4 \%$ vs $81.1 \%, \mathrm{p}<0.001)$. This continued to 1 -year $(48.5 \%$ vs $68.2 \%$, p $<0.001)$. Frailty significantly increased mortality hazards in covariate- adjusted analyses at 30-days (HR 1.56; 95\%CI 1.14-2.15; $\mathrm{p}=0.006$ ), and 1-year (HR 1.35; 95\%CI 1.03-1.76; $\mathrm{p}=0.028$ ). Singlepoint CFS increases were associated with a 30 -day mortality hazard of $1.23(95 \% \mathrm{CI} 1.13-1.34 ; \mathrm{p}<0.001)$ in unadjusted analyses, and $1.11(95 \% \mathrm{CI} 1.01-1.22 ; \mathrm{p}=0.026)$ after covariate adjustment. Frail patients received significantly more days of LST (median[IQR]: 5[3,11] vs 4[2,9], p=0.008).

Discussion: Frailty was significantly associated with greater mortality at all time points studied, but most notably in the first 30- days post-ICU admission. This was despite greater LST use. The accrual effect of frailty increased adverse outcomes.Point-by-pointuseoffrailtyscoringcouldallowformoreinformeddecisionmakinginICU.

Conclusions: *See above-Discussion/Conclusions combined*

\section{Co-Creation and Evaluation of a "Sustain and Gain" Falls Prevention Maintenance Program for Community- Dwelling Older Adults in Greater Sudbury}

Benoit Lafleur ${ }^{1}$, Samantha Jibb ${ }^{2}$, Shaen Gingrich ${ }^{2}$, Susan Lane ${ }^{2}$, Jo-Anne Clarke ${ }^{2}$

${ }^{1}$ Northern Ontario School of Medicine, ${ }^{2}$ North East Specialized Geriatric Centre and Health Sciences North

Background: Group-based fall-prevention exercise programs reduce the rate of falls amongst community-dwelling older adults. Participants in our local falls program improved their balance performance upon completion, but these benefits were lost after a 6-month follow-up. The goal of this study is to test the efficiency of 'Sustain and Gain', a pilot program designed to continue the improvement in balance performance in graduates of the fall program.

Methods: The 'Sustain and Gain' program was developed in two phases. Phase I (developing the maintenance program) used an experienced-based co-design that included 11 participants, their care partners, and members of the research team. The program was decided to be biweekly one-hour balancefocused exercise sessions for 9 months, with a half-hour educational component per week. Phase II (implementation and evaluation) used a mixed methodology that included: quantitative analysis to measure the participants' physical outcomes (Timed Up and Go, 2-minute walk test, Berg Balance Scale) and quality of life (Older Peoples' Quality of Life Questionnaire) measured at baseline, 3 months, 6 months, and 9 months, as well as a qualitative analysis to gain a deeper understanding of participants' experience and their satisfaction with the program.
Results: Eleven participants completed the full program and three participants completed months three to nine. The mean (SD) age was 78.6 (5.78) years. There was a general improvement in balance performance at nine months, and qualitative analyses demonstrated positive feedback about the program.

Discussion: The 'Sustain and Gain' program may continue the improvement of balance performance of frail older adults. The positive social and psychological outcomes expressed by the participant's further support the benefit of the program.

Conclusions: Participants benefited from the 'Sustain and Gain' program.

\section{Adverse events in alternate-level-of-care patients awaiting long-term-care in hospital}

Guillaume Lim Fat ${ }^{1}$, Aquila Gopaul ${ }^{2}$, Demetri Pananos ${ }^{1}$, Mary-Margaret Taabazuing ${ }^{1}$

${ }^{1}$ Western University, ${ }^{2}$ University of Calgary

Background: Inrecentyears,agrowingnumberofelderlypatie ntsareawaitinglong-termcare(LTC)placementinhospitalwhile designated Alternate-Level-of-Care (ALC). In addition to infrastructural strain created by occupying acute care beds, ALC patients also incur healthcare-associated adverse events during their prolonged hospitalization. Our observational studydescribesratesofadverseeventsinALCpatientsawaitingLTCinacutecarehospitals.

Methods: Our chart review included a random sample of 156 patients of 2386 ALC-designated patients who were awaiting LTC at two tertiary-care centres between 2015 and 2018. Incidence of adverse events was collected, including healthcare-associated infections, falls, delirium, pressure ulcers, and venothrombotic events. We used a restricted cubic spline to model the expected number of adverse events as a function of length of ALC stay.

Results: We recorded 362 total adverse events occurring over 8,668 ALC days. ALC patients waited an average of 55 days before LTC placement, with 7 deaths occurring prior to placement. Our model shows an expected 1.08 adverse events at 14 ALC-days, 1.81 at 30 ALC-days, and 2.93 at 60 ALC-days. The most common infectious adverse events were urinary tract infections (53.2\%) and respiratory infections (27.6\%). A total of 620 additional antimicrobial days were prescribed as a result of such infections. The most common non-infectious adverse events were delirium (28.3\%) and falls (14.6\%).

Discussion: Our findings describe a substantial burden of adverse events in ALC patients and suggest that waiting in hospital for LTC infers a quantifiable risk which increases predictably with length of stay.

Conclusions: We aim to use this data to educate patients and caregivers regarding risk for healthcare-associated adverse events, and advocate for improved LTC infrastructure and access with prospective strategies to reduce ALC stays. 


\section{Impact of Dementia on Outcomes in a Seniors Falls Prevention Program}

Lindsay Schnarr ${ }^{1}$, Marilyn Bater ${ }^{2}$

${ }^{1}$ University of British Columbia, ${ }^{2}$ Royal Jubilee Hospital

Background: Elderly individuals with dementia have a significantly higher falls risk than those with normal cognition. Studies have demonstrated the effectiveness of exercise for falls prevention. The purpose of this study was to analyze data on participants in a falls prevention program to determine if dementia impacted intervention efficacy.

Methods: Retrospective chart review analyzing pre-and postintervention data was performed for participants in the Falls Prevention Program at Royal Jubilee Hospital (Victoria, BC). Subjects $(n=167)$ had a documented MOCA score and pre-and post-program scores for at least one of five tests (Berg Balance Scale, gait velocity preferred, gait velocity fast, timed up-and-go, 30-second sit-to-stand). Paired t-tests and ANOVA tests were used to analyze pre-and post- scores based on MOCA result.

Results: Participants without dementia $(\mathrm{MOCA}=26+)$ demonstrated improvement in scores for all 5 tests $(\mathrm{p}<0.001)$. Those with mild dementia (MOCA=18-25) improved in all except for gait velocity preferred $(\mathrm{p}<0.05)$. The moderate dementia group $(\mathrm{MOCA}=10-17)$ only showed improvement for Berg Balance Scale $(\mathrm{p}<0.001)$. No participants had severe dementia $(\mathrm{MOCA}<10)$. When comparing the interval change in scores between those with dementia $(\mathrm{MOCA}<26)$ and those without $(\mathrm{MOCA}=26+)$ there was only a significant difference identified in timed up-and-go scores ( $\mathrm{p}<0.05)$.

Discussion: Scores on tests indicative of falls risk and frailty improved after participation in a falls prevention program for those with mild dementia but not moderate dementia, demonstrating benefit in the mild group. No difference in improvement of scores was seen when comparing those with normal cognition versus any severity of dementia, leading us to believe that these two groups derived similar benefit from the program.

Conclusions: Falls prevention programs appear to be effective in those with mild dementia and should be used to try and reduce morbidity and mortality.

\section{ELIGIBLE FOR THE DR. JACK \& DR. ASA AWARD}

\section{Development of an Evidence-Based Reminder Tool to Improve Care For Older Adults During Periodic Health Examinations.}

Serina Dai, Amy Freedman

University of Toronto

Background: Guidelines for prevention and health maintenance have become more complex as the population ages.
Delivery of these maneuvers is often challenging given the constraints of general practice. Evidence based reminder tools for younger populations have been validated and widely adopted in primary care. The objective of this project is to develop an evidence based, patient centered reminder tool, available in paper and electronic format, to help primary care providers deliver interventions that have been shown to improve function, quality of life or mortality in older adults.

Methods: The Ovid MEDLINE database was searched using specified key words and other terms relevant to preventative health care and guidelines on primary health care and the elderly. Articles were limited to English-language publications between 2010 and 2020. Secondary sources, such as the Canadian Task Force on Preventive Health Care, the Public Health Agency of Canada, and the Canadian Medical Association Infobase, were also searched.

Results: A checklist form covering recommended health care maneuvers was developed in both paper and electronic format. The form incorporates recent evidence-based preventive care guidelines and research on preventive care for older adults. The strengths of the recommendations (good, fair, inconclusive) are indicated in the tool. A section of the tool is specific to the frail older adults and those with dementia. Links to additional resources are embedded in the tool.

Discussion: Primary care providers can use this tool to guide preventive screening and chronic disease management in older adults. Assessment of the tool's efficacy is needed. The tool will need to go through a peer review process prior to widespread dissemination in primary care.

Conclusions: This low-cost, easy-to-use tool has the potential to improve the delivery of evidence-based care to older adults during period health examinations.

\section{Premorbid Functional Status as a Predictor of Discharge Destination in Older Adults Hospitalized For COVID-19: A Cohort Study}

Sophie Del Degan, Marie-France Forget, Julie Leblanc, Michaël Desjardins, Madeleine Durand, Thien Tuong Minh $\mathrm{Vu}$, Quoc Dinh Nguyen, Philippe Desmarais Centre Hospitalier de l'Université de Montréal (CHUM)

Background: Functional outcomes and discharge destination of older adults hospitalized for coronavirus disease 2019 (COVID-19) have not been well described. Identification of factors associated with favourable post-hospital functional outcomes could aid with care planning and decision-making. This study aimed to describe the functional trajectories of older adults hospitalized with COVID-19 and to identify potential predictive factors for in-hospital functional recovery.

Methods: This cohort study of adults aged 65 and older with a COVID-19 diagnosis took place in an academic healthcare institution between April and May 2020. COVID-19 was diagnosed by a positive nasopharyngeal swab. Katz scores were 
computed as a marker of premorbid functional independence. The primary outcome was patient's discharge destination, demonstrating either functional status stability (i.e., return home) or declining functional status (i.e., discharge to a rehabilitation centre, institutionalization, or death).

Results: A total of 96 community-dwelling older adults were hospitalized with a diagnosis of COVID-19. The median age was 81 years (IQR: 74-87), with 41(43\%) females. Before hospitalization, 41 (43\%) patients were living in private housing, 32(33\%) in seniors' housing, and $21(22 \%)$ in assisted living residence. Overall, 34(35\%) patients died, 26(27\%) were discharged to a rehabilitation centre, or institutionalized, and 36(38\%) to their pre-hospital living.

Premorbid functional independence in activities of daily living was associated with an increased probability of being discharged to the pre-hospital living situation (OR: 4.3; 95\%CI: 1.6-11.4).

Discussion: Our results are exploratory because of the small sample size. Premorbid functional independence is a predictive factor of discharge destination, but no associations were identified for age, sex, and medical comorbidities.

Conclusions: In this cohort of older adults with COVID-19, about one third of patients were discharged home following hospitalization. Premorbid functional independence can guide with care planning.

\section{Understanding Older Adults' Experiences with Telemedicine During the COVID-19 Pandemic: An Exploration of Barriers, Facilitators, and Quality Indicators.}

Amy Miles, Mireille Norris

University of Toronto

Background: The COVID-19 pandemic required rapid adoption of telemedicine to deliver care to older adults. Older adults and their caregivers report high rates of satisfaction with telemedicine care in quantitative surveys, but survey data does not capture gaps in care or issues in need of improvement. We aimed to better understand older adults' perceptions of receiving telemedicine care and identify areas for improvement.

Methods: This study used qualitative methodology. Individual interviews were conducted over the telephone with older adult patients of the Sunnybrook HSC Geriatric Medicine outpatient clinics. Patients and caregivers who received telemedicine care from March 1 to Dec 31, 2020, either over the telephone or using video-conferencing technology, were invited to participate in a 30-minute interview to explore their perceptions of the telemedicine experience. Interviews were audio-recorded and transcribed verbatim. Data was analyzed using thematic analysis.

Results: Eight older adults and their care partners participated in interviews. All participants perceived a positive telemedicine experience and reported feeling open to telemedicine as an option for future care. Four dominant themes were identified and explored: the role of self, the role of technology, the role of the physician, and the role of care partners in telemedicine. These themes illustrate how health goals can be addressed and facilitated through telemedicine.

Discussion: Telemedicine involves complex interactions between patient, provider, caregiver, and technology. Patients should be placed at the centre of this interaction to ensure that their needs are met. These results are expected to form the basis for future quality improvement interventions aimed at improving equity and effectiveness of telemedicine for older adults.

Conclusions: Telemedicine was perceived as an effective and useful modality to address health issues in older adults.

\section{Worries, Attitudes, and Mental Health Symptoms of Older Adults during the COVID-19 pandemic: Canadian and US Perspectives}

Christina Reppas-Rindlisbacher ${ }^{1}$, Jessica M Finlay ${ }^{2}$, Alyson Mahar ${ }^{3}$, Shailee Siddhpuria ${ }^{4}$, Julie Hallet ${ }^{1}$, Paula Rochon $^{1}$, Lindsay C Kobayashi ${ }^{2}$ ${ }^{1}$ University of Toronto, ${ }^{2}$ University of Michigan, ${ }^{3}$ University of Manitoba, ${ }^{4}$ University of British Columbia

Background: Differences in older adults' worry, attitudes, and mental health between high-income countries with diverging pandemic responses are unknown. We compared COVID-19 worry, attitudes towards government responses, and selfreported mental health symptoms among adults aged $\geq 55$ in the US and Canada early in the COVID-19 pandemic.

Methods: We administered an online cross-sectional survey between April 2nd and May 31st in the US and between May 1st and June 30th, 2020 in Canada to a convenience sample of older adults aged $\geq 55$. Likert-type scales measured COVID-19 worry and attitudes towards government support. Three standardized scales assessed mental health symptoms: the eight-item Center for Epidemiological Studies Depression Scale, the five-item Beck Anxiety Inventory, and the threeitem UCLA loneliness scale.

Results: There were 4453 US respondents $(71.7 \%$ women; mean age 67.5 (7.5)) and 1549 Canadian (67.6\% women; mean age 69.3 (7.8)). More US respondents (71\%) were moderately or extremely worried about the COVID-19 pandemic, compared to $52 \%$ in Canada. Just $20 \%$ of US respondents agreed or strongly agreed that the federal government cared about older adults in their pandemic response, compared to $63 \%$ of Canadians. US respondents were more likely to report depressive and anxiety symptoms compared to Canadians; $34.2 \%(32.8-35.6)$ vs. $25.6 \%(23.3-27.8)$ for depressive and $30.8 \%$ (29.5-32.2) vs. $23.7 \%$ (21.6-25.9) for anxiety. The proportion of respondents who reported loneliness in both countries was similar.

Discussion: US older adults felt less supported by their federal government and had a higher proportion of depressive and anxiety symptoms compared to older Canadians during the early months of the COVID-19 pandemic. 
Conclusions: Public health messaging in both countries should focus on supporting the mental health needs of older adults, and communication from governments should be clear, consistent, and apolitical.

\section{Researching the Effects of sleep on STep count dUring the Post-operative period (REST-UP)}

Christina Reppas-Rindlisbacher ${ }^{1}$, Manan Ahuja ${ }^{2}$, Eric Wong ${ }^{1}$, Jessica Gormley ${ }^{2}$, Shailee Siddhpuria ${ }^{3}$, Justin Lee ${ }^{2}$, Maeve Conroy ${ }^{3}$, Christopher Patterson ${ }^{2}$ ${ }^{1}$ University of Toronto, ${ }^{2}$ McMaster University, ${ }^{3}$ University of British Columbia

Background: Hospitalized older adults commonly experience functional decline and sleep deprivation. Our aim was to determine whether total sleep time measured by an activity monitor predicts step count in older adults with hip fractures.

Methods: This was a prospective observational cohort study of older adults ( $>65$ years) with hip fractures admitted to an academic hospital in Hamilton, Ontario between Jan 2018 - June 2019. Fitbit ${ }^{\circledR}$ devices were used post-operatively to measure total sleep time and step count. Mobility was defined as mean daily steps and meters walked by physiotherapy notes. We used a multivariate regression analysis to evaluate the association between total sleep time and number of steps. The associations between sleep and other predetermined outcomes of interest such as delirium were also tested.

Results: Our final analysis included 62 participants [mean age (SD) 82.3 (8.4], 67.7\% women]. Mean number of steps and meters walked increased from 458 steps and 9.8 metres on Day 1 to 610 steps and 31 metres on Day 10. On average, participants slept 5.4 hours and experienced 5.3 awakenings per night. Increased mean total sleep time was not associated with more steps walked (unadjusted $\beta=-0.21 ; 95 \% \mathrm{Cl}$ $0.28-10.75$, $p$ value $=0.46$ ). $33 \%$ of participants experienced post-operative delirium. Increased mean total sleep time was associated with a decreased risk of delirium $(\mathrm{aOR}=0.66 ; 95 \%$ $\mathrm{Cl}$ 0.46-0.88, $\mathrm{p}$ value 0.012 ).

Discussion: Older adults recovering from hip fracture surgery experience poor sleep with multiple disruptions per night. Though sleep time was not associated with improved step count, every 1 hour increase in sleep time decreased the odds of delirium by $34 \%$.

Conclusions: Addressing poor sleep during hospitalization may reduce the risk of post-operative delirium in older adults with hip fractures.

\section{A Qualitative Study of Patient and Caregiver Experiences Using a Mobile Application to Access Virtual Care for Homebound Older Adults}

Victoria YY Xu${ }^{1}$, Rosalind Sham², Sally $\mathrm{Moy}^{2}$, Gary Naglie $^{2}$, Jagger Smith ${ }^{2}$

${ }^{1}$ University of Toronto, ${ }^{2}$ Baycrest
Background: This qualitative study aims to explore patient/ caregiver experiences using a mobile application (Akira), as part of a pilot virtual care service for homebound older adults receiving in-home care through Baycrest's Integrated Community Care Team. Patients/caregiver dyads were given a smartphone with Akira, which provides access to text/ video chat with a nurse practitioner (NP) at all hours. Dyads consisted of the person who consented to research participation (patient/substitute decision maker) and primary app user (patient, family caregiver or paid caregiver).

Methods: From May to August 2020, semi-structured phone interviews were conducted with 27 app users (4 ICCT patients, 6 paid caregivers and 17 family caregivers). The audio-recorded interviews were transcribed, verified for accuracy and anonymized. Thematic analysis was conducted with line-by-line coding using NVivo v11, with the use of a double-coder.

Results: Qualitative interviews revealed key concepts influencing participants' experiences. Participants used the app for various health concerns. Those who did not use the app either did not need it or used alternate healthcare resources. The main facilitators were the app's ease of use and responsiveness of Akira NPs, and barriers included insufficient training and Akira's logistical limitations. While participants reported initial hesitation/skepticism towards using Akira, they were reassured about access to after-hours healthcare. Participants also spoke favourably towards the convenience and value of having Akira during the COVID-19 pandemic.

Discussion: Our study uniquely focuses on the experiences of homebound older adults and their caregivers, with a mobile app integrated into a home-visiting care team. Limitations include small sample size for patient and private caregiver subgroups.

Conclusions: Participants' feedback will inform program improvements. Further research is needed to explore the role of telemedicine in the care of homebound older adults.

\section{ELIGIBLE FOR THE EDMUND V. COWDRY AWARD}

The Effect Of A Multimodal Exercise Intervention
Combined With Computerized Cognitive Training
On Depressive Symptoms In Older Adults With
Mild Cognitive Impairment: A Preliminary Report

Joel Mahon ${ }^{1}$, Quincy Almeida ${ }^{2}$, Louis Bherer ${ }^{3}$, Amer Burhan $^{4}$, Teresa Liu-Ambrose ${ }^{5}$, Laura Middleton ${ }^{6}$, Lindsay Nagamatsu $^{1}$, Mark Speechley ${ }^{7}$, Manuel Montero-Odasso ${ }^{8}$ ${ }^{1}$ Department of Kinesiology, Western University, ${ }^{2}$ Sun Life Financial Movement Disorders Centre, Wilfred Laurier University, ${ }^{3}$ Faculty of Medicine, University of Montreal, ${ }^{4}$ Department of Psychiatry, University of Toronto, ${ }^{5}$ Department of Physical Therapy, University of British Columbia, ${ }^{6}$ Department of Kinesiology, University of 
Waterloo, ${ }^{7}$ Department of Epidemiology and Biostatistics, Western University, ${ }^{8}$ Gait and Brain Lab, Parkwood Institute and Lawson Health Research Institute

Background: Depressive symptoms (DS) are common in older adults with mild cognitive impairment (MCI) and confer a higher likelihood of progression to dementia. Two interventions that may reduce DS include physical exercise and computerized cognitive training (CCT). Currently, the impact of combining multimodal exercises with CCT programs on DS in older adults with MCI is unclear. Our objective was to evaluate whether a 5-month program combining exercise and CCT would result in a greater reduction of DS than either multimodal exercise or control groups alone, in older adults with MCI.

Methods: Data is obtained from the SYNERGIC trial, a RCT for this secondary analyses. 68 participants ages $\geq 60$ were randomized to one of three groups; Group 1: multimodal exercise and $\mathrm{CCT}(\mathrm{N}=27)$, Group 2: multimodal exercise and sham CCT $(\mathrm{N}=26)$, and Group 3: control group performing stretching exercises and sham CCT $(\mathrm{N}=15)$. All groups attended intervention sessions 3-times weekly. The Geriatric Depression Scale-30 (GDS-30) was used to measure DS at baseline and post-intervention. Two-way repeated measures ANOVA was used to evaluate the effect of different treatments on DS.

Results: Mean age was $74.30 \pm 5.88,52.9 \%$ female. Following intervention GDS-30 scores decreased by $20.62 \%$ and $4.08 \%$ forGroups 1and2respectively, while increasingby $11.91 \%$ inthea ctivecontrolgroup $(\mathrm{p}=0.150)$. Theinteraction between time and treatment became significant ( $\mathrm{p}=0.018$ ) when using stratification analysis byGDS-30 $>9$.

Discussion: Our findings suggest this multimodal exercise and cognitive training intervention may be a viable option for reducing DS in older adults with MCI, especially in those with GDS-30>9.

Conclusions: A 5-month intervention combining multimodal exercise with cognitive training significantly reduced DS in older adults with MCI when compared with exercise training alone or control exercise.

\section{Fall Risk-Increasing Drugs (FIRDS) and Gait Performance in Community-Dwelling Older Adults: Results from the Gait and Brain Study}

Abdelhady Osman ${ }^{1}$, Mark Speechley ${ }^{1}$, Shehzad Ali ${ }^{1}$, Manuel Montero-Odasso ${ }^{2}$

Department of Epidemiology and Biostatistics - Western

University, ${ }^{2}$ Gait and Brain and Lab-Parkwood Institute

Background: Several psychotropic and cardiovascular drugs have been identified as fall risk-increasing drugs (FRIDS) in older adults. The mechanisms linking between FRIDS and falls are unclear. It is plausible that gait is on the causal pathway between FRIDS and falls. Additionally, there is a knowledge gap on the association between cardiovascular FRIDS and gait performance. We aimed to assess the association between FRIDS and gait performance parameters including speed and gait variability in community-dwelling older adults.

Methods: This was a cross-sectional analysis using data from the Gait and Brain Study, a study of community-dwelling older adults aged 65 years old and over without neurological disorders $(\mathrm{N}=345)$. Medication use was ascertained through validated questionnaires and electronic medical records. Separate multiple linear regression models were created to assess the association between each drug class and gait speed and gait variability. Gait variability was measured as the coefficient of variation $(\mathrm{CoV})$ of stride time. The following drug classes were assessed: antidepressants, benzodiazepines, alpha-blockers, beta-blockers, vasodilators, diuretics, statin and aspirin.

Results: After adjustment for potential confounders, diuretic use was associated with significantly reduced gait speed (Beta $=-7.94,95 \%$ CI: $-13.9,-1.98, \mathrm{P}=0.009$ ). Statin use was associated with significantly increased gait variability (Beta= 0.11, 95\%CI: 0.01, 0.23, $\mathrm{P}=-0.04)$.

Discussion: The association between diuretic use and reduced gait speed is consistent with existing evidence on diuretic use and increased fall risk. The association between statin and increased gait variability, a marker of gait instability and increased fall risk, is notable given the null associations reported between statin and gait speed in previous studies.

Conclusions: Diuretic use and statin use are associated with reduced gait performance in community-dwelling older adults. Further longitudinal research is required to establish causality.

\section{Medication Optimization Intervention and Potentially Inappropriate Medications: What Is The Impact in Real Life?}

Anaïs Payen ${ }^{1}$, Jean-Baptiste Beuscart ${ }^{1}$, Julien Soula ${ }^{1}$, David Verloop ${ }^{2}$, Marguerite-Marie Defebvre ${ }^{2}$, Delphine Dambre ${ }^{3}$, Claire Godard-Sebillotte ${ }^{4}$

${ }^{I}$ Univ. Lille, CHU Lille, ULR 2694 - METRICS : Évaluation des technologies de santé et des pratiques médicales, F-59000 Lille, France, ${ }^{2}$ Agence Régionale de Santé Hautsde-France, Lille, France, ${ }^{3}$ Service médecine polyvalente, Centre Hospitalier de Saint-Amand-les-Eaux, France, ${ }^{4}$ Department of Family Medicine, McGill University, Montreal, Canada

Background: Potentially inappropriate medications (PIMs) are highly prevalent in older people up to $60 \%$. Medication optimization has been shown to reduce PIMs' prevalence. The PAERPA (Personnes Âgées En Risque de Perte d'Autonomie) intervention included an in-hospital medication optimization intervention. This intervention was implemented in a real-life context outside of the controlled conditions of a randomized experiment. The aim was to evaluate the impact of the medication optimization intervention on PIMs. 
Methods: We analyzed a retrospective cohort of older persons enrolled in the PAERPA intervention between January 1st, 2015 and December 31st, 2018 in the ValenciennoisQuercitain area in France. The control group was built by extracting, from the French health administrative database, similar persons, hospitalized during the same period in an equivalent general hospital, but not having received the in-hospital intervention. We defined PIMs with the French Laroche list, the STOPP list and the European PIM list. We compared the intervention and control group in terms of PIMs by using the difference-in-difference analysis.

Results: The 582 persons enrolled in the study had an average age of $82.9 \pm 4.9$ years, $65.3 \%$ were women. The control and intervention groups had the same socio-demographic, clinical and drugs characteristics (including median number of PIMs). For the both group and depending on the list used, the median number of PIMs ranged between $2[0 ; 3]$ at the hospital admission and $3[0 ; 3]$ at discharge. There was no significant difference in the mean number of PIMs associated with the intervention.

Discussion: Even though medication optimization interventions have shown promising results in a controlled randomized context, there impact might be less conclusive in real-life implementation.

Conclusions: We will conduct further analysis to understand the factors that can explain this absence of effect.

\section{Dual-Task Gait Cost and Cerebrovascular Lesion Burden In Older Persons: Results from ONDRI.}

Frederico Pieruccini-Faria ${ }^{1}$, Seyyed Haddad ${ }^{1}$, Ben Cornish ${ }^{2}$, Bill McIlroy ${ }^{2}$, Rob Bartha ${ }^{1}$, All ONDRI Investigators $^{3}$, Manuel Montero-Odasso

${ }^{1}$ University of Western Ontario, ${ }^{2}$ University of Waterloo, ${ }^{3}$ Ontario Brain Institute

Background: The slowing of walking speed caused by a cognitive task or the dual-task gait cost (DTC) is a predictor of dementia and physical disability in older individuals who sustain cerebrovascular lesions. However, whether and how the DTC is associated with the global and local cerebrovascular lesion burden in older persons remains unclear. We investigatedtheassociationbetweenDTCandthecerebrovascularlesionburdenrepresentedbythepercentageof white matter hyperintensity volume in different brainregions.

Methods: $A$ total of 123 participants (69.8-7.31 years of age; $32 \%$ femal) with history of cerebrovascular disease or stroke from the Ontario Neurodegenerative Disease Research Initiative (ONDRI) were analyzed. The cerebrovascular lesion burden was calculated in the frontal lobe, parietal lobe, temporal lobe, occipital lobe and basal- ganglia +thalamus region. Gait speed was electronically assessed during self-paced (single-task) and while naming animals (dual-task) conditions. DTC was the percentage of slowing from single to dual-task condition. The multivariate linear regression model tested the association between the DTC and cerebrovascular lesion burden in different brain locations, adjusted for age, sex, years of education and the national institute health stroke score.

Results: DTC and the overall cerebrovascular lesion burden were significantly associated (Wilk's lambda $=.88$, $\mathrm{p}=.01$; eta $2=.111$ ) with a significant contribution of the cerebrovascular lesion burden in basal- ganglia+thalamus $($ Beta $=.009 ; \mathrm{p}=.003 ; \mathrm{eta} 2=.070)$.

Discussion: White matter lesion burden, particularly in subcortical regions, where sensory and automatized motor processes are modulated, may play an important role on dualtasking difficulties while walking in patients with history of cerebrovascular lesions.

Conclusions: Gait in patients with history of cerebrovascular lesions may be more cortically controlled as a compensation for impaired subconscious/automatic motor control processes.

\section{In Critically Ill Older Patients, Mobility, Not Illness Severity, Is Associated With 30-Day Mortality}

Samuel Searle ${ }^{1}$, Steve Walsh ${ }^{2}$, Giselle Davis ${ }^{3}$, Tara Mercier ${ }^{3}$, Kenneth Rockwood ${ }^{4}$, Daniel Davis ${ }^{2}$, Babar Haroon ${ }^{4}$

${ }^{I} M R C$ Unit Lifelong Health and Ageing at UCL, University College London/Dalhousie University, ${ }^{2}$ Health PEI, ${ }^{3}$ Nova

Scotia Health Authority, ${ }^{4}$ Dalhousie University

Background: Critical illness accelerates frailty development. Understanding health trajectories, of older critically ill patients, is needed to determine interventions for frailty. Here we investigate the relationship between illness severity, frailty and mobility trajectory on 30-day survival and also determine the prognostic benefit of early in-hospital mobility improvement for critically ill patients.

Methods: This is a prospective cohort study of older adults $(>64)$, admitted to two intensive care units (ICU). Baseline demographics, Sequential Organ Failure Assessment (SOFA) score, and Clinical Frailty Scale (CFS) was obtained upon ICU admission. Mobility was obtained daily in ICU, using the Hierarchical Assessment of Balance and Mobility (HABAM). A joint model: random effect model for HABAM trajectory and cox proportional hazards model for 30- day mortality was used to determine the relationship between frailty (CFS), illness severity (SOFA score), mobility (HABAM) and mortality.

Results: The cohort consisted of 86 mobilized intensive care unit participants. The mean age and SOFA Score were 72.3 and 8.0, respectively. The median CFS was 4 . In the joint model, increasing CFS was associated with lower HABAM scores $(-0.90, p=0.01)$, and attenuated daily HABAM improvements $(-0.22, p=0.02)$. SOFA Score was not associated with 30-day mortality $(\mathrm{HR}=1.16, \mathrm{p}=0.12)$ but HABAM trajectory $(\mathrm{HR}=0.18, \mathrm{p}=0.03)$ was. Early ICU improvements in 
the HABAM predicted hospital survival (Sensitivity $=77 \%$, Specificity $=63 \%$, AUC $=0.7$ ).

Discussion: Mobility trajectory, not SOFA Score, is associated with 30-day mortality. The relationship demonstrated between mobility and frailty here is consistent with the literature. Mobility, and other frailty related recovery measures, need to be investigated further for their utility in the identification of high-risk patients for intervention and the timely communication of patient progress.

Conclusions: Mobility trajectory, not illness severity, is associated with 30-day mortality in critically ill older patients.

\section{Virtual Reality Exposure Therapy Reducing Responsive Behaviors in Nursing Home Residents with Dementia: A Case Series}

Munira Sultana ${ }^{1}$, Karen Campbel12 , Morgan Jennings ${ }^{2}$, Joseph B. Orange ${ }^{2}$, Jill Knowlton ${ }^{3}$, Armin St. George ${ }^{4}$, Manuel Montero-Odasso ${ }^{1}$, Dianne Bryant ${ }^{2}$

${ }^{I}$ Gait and Brain Lab, Parkwood Institute and Western

University, ${ }^{2}$ Western University, ${ }^{3}$ Primacare Living

Solutions Inc.TM, ${ }^{4}$ Crosswater Digital Media, LLC

Background: People with advanced dementia often exhibit responsive behaviors such as apathy, depression, agitation, aggression, sleep disorders, and psychosis. Responsive behaviors can cause physical and emotional harm to the individual and their caregivers. Non-pharmacological approaches (e.g. listening to music, watching television, arts and crafts) are now a first-line strategy to manage responsive behaviours in clinical practice because of potential risks associated with the use of antipsychotic medications. This study evaluates whether virtual reality exposure therapy is a feasible nonpharmacological intervention to reduce responsive behaviors in nursing home residents with advanced dementia.

Methods: We conducted a single site case series with a convenience sample $(\mathrm{N}=24$; age $=85.8 \pm 8.6$ years; Cognitive Performance Scale score $=3.4 \pm 0.6$ ). Our intervention was 30 minutes of customized immersive three-dimensional virtual reality delivered using remote-controlled projectors for five days a week for two weeks. Outcomes included the Cornell Scale for Depression in Dementia (CSDD) and Cohen-Mansfield Agitation Inventory (CMAI) score agitation pre-and-post-intervention.

Results: The intervention was feasible with $0 \%$ attrition. The average length of each session was 22 minutes. Eight out of 24 of participants were able to complete $80 \%$ of the sessions. No related adverse events were registered. A clinically significant reduction in depression scores $(\mathrm{ES}=0.4, \mathrm{p} \leq 0.05)$ and in agitation scores $(\mathrm{ES}=0.2, \mathrm{p}<0.06)$ was observed post-intervention.

Discussion: Our intervention is remote-controlled, does not need a headset, and is compatible with COVID-19 infection prevention policies such as physical distancing, disinfection routine, and social isolation; promising a novel approach to reduce responsive behaviors among the nursing home residents. A future RCT can confirm our results.

Conclusions: Virtual reality exposure therapy is a safe and feasible non-pharmacological intervention improves responsive behaviors in nursing home residents with dementia

\section{POSTERS}

\section{COVID-19 Outcome: Comparison Between Older and Younger Adults}

Ripa Akter ${ }^{1}$, Kristine Kim ${ }^{2}$, Allen Huang ${ }^{2}$

${ }^{I}$ The Ottawa Hospital, ${ }^{2}$ The Ottawa University

Background: Frail older adults with underlying chronic medical conditions are at a higher risk of contracting COVID-19 infection, presenting atypically and developing severe complications. We aim to examine and compare presentations and outcomes between older and younger adults.

Methods: A retrospective observational cohort study was performed analyzing data (baseline characteristics, clinical presentation, and complications) from The Ottawa Hospital COVID-19 registry from February 1, 2020 to June 30, 2020 in patients $<65,65-79$ and $80+$ years. Chi-Square Test or Fisher's Exact Test for categorical variables, and Kruskal-Wallis Test and Wilcoxon rank-sum test to compare median were used.

Results: There were a total of 127 patients ( 55 were aged $<65$, 38 were $65-79,34$ were $80+$ ). Nearly $20 \%$ had baseline dementia with associated higher length of stay (LOS) $\mathrm{P}<0.05$ and trend towards higher delirium rates $(\mathrm{P}=0.06)$. Hypertension, coronary artery disease, active cancer were more frequent in $65+$, whereas obesity and chronic lung disease in $<65$. Adults $<65$ presented with typical respiratory illness and more likely to be admitted to ICU compared to older adults who presented with weakness and confusion with higher rates of death, delirium and increased median LOS $(\mathrm{P}<0.05)$. Older adults had higher Median CRP, D-Dimer, Urea, and lower white cell count/lymphocytes. Common imaging findings in 65-80+ combined were either patchy, diffuse, reticular, or ground glass opacity, bilateral involvement, and less commonly clear chest imaging.

Discussion: Our study supports existing evidence and provides further detailed evidence into atypical COVID-19 presentations and outcomes in older adults. A possible explanation for higher mortality in older adults includes pre-existing goals of care or resource allocation limitations.

Conclusions: Older adults with COVID-19 commonly present with atypical symptoms and have worse outcomes. 


\section{What Are The Differences in Health Service Use Between Persons With Dementia Living in Rural and Urban Areas In Quebec?}

Geneviève Arsenault-Lapierre ${ }^{1}$, Tammy Bui ${ }^{1}$, Claire Godard-Sebillotte ${ }^{1}$, Nadia Sourial ${ }^{2}$, Amélie QuesnelVallée $^{1}$, Victoria Massamba ${ }^{3}$, Louis Rochette ${ }^{3}$, Debra Morgan $^{4}$, Julie Kosteniuk ${ }^{4}$, Isabelle Vedel ${ }^{1}$

${ }^{1}$ Mc Gill University, ${ }^{2}$ Université de Montréal, ${ }^{3}$ Institut national de santé publique du Québec, ${ }^{4}$ University of Saskatchewan

Background: Patients with dementia have higher healthcare utilization than patients without, but little is known about how rurality modulates this relationship. Rural patients are more likely to face barriers in obtaining health care, such as experiencing longer travel times, delayed referrals to specialists, and uneven healthcare resources compared with urban patients. The aim of this study is therefore to describe healthcare service utilization between rural and urban patients with dementia in Quebec.

Methods: We used a repeated annual cross-sectional cohort design from 2000 to 2019, with linked administrative databases from the Institut national de santé publique du Québec. All incident cases of dementia in community-dwelling persons $65+$ occurring between April 1st and March 31st for each year were included. Dementia diagnosis was ascertained using a validated algorithm. Rurality was defined by Statistical Area Classification (SAC) types.

Results: Overall, we present age-adjusted trends for 237259 patients with dementia. Of these people, $79,8 \%$ live in urban areas (SACtypes 1 to 3), while $20,2 \%$ live in rural areas (SACtypes 4 to 7). Graphical and visual analyses revealed that rural PWD have more emergency department visits, more hospitalizations too, but stay the shortest in hospital and are sent less to alternate level of care. Rural PWD also have fewer visits to primary care physicians and cognition specialists. Rural and urban PWD have similar rates of long-term care admission and mortality.

Discussion: This study reveals important geographically based differences in health service use between rural and urban patients with dementia in Quebec.

Conclusions: These results are important as geographical disparities need to be addressed in dementia strategies and interventions, otherwise health inequity may be exacerbated. This research will guide researchers and decision- makers to develop more equitable health policies.

\section{The Geriatrician's Perspective on Attending A Former Patient's Funeral: a qualitative study.}

Vincent Aubin ${ }^{1}$, Steve Paquet ${ }^{2}$, Pierre J. Durand ${ }^{1}$, Félix Pageau ${ }^{1}$

${ }^{1}$ Université Laval, ${ }^{2}$ Centre d'excellence sur le vieillissement de Québec
Background: The aim of our study was to better understand the perspective of the medical geriatrics specialist on attending a deceased patient's funeral. Our secondary goals were to better (1) define the limits of professionalism and therapeutic relationships, (2) understand the factors that influence a geriatrician's presence at a former patient's funeral and (3) identify potential beneficial effects of this custom.

Methods: Using an exploratory qualitative method, we conducted two focus group interviews with, in total, ten geriatricians from Quebec City. Each group discussion explored the physician's personal experiences and professional perspectives on the matter, including ethical and deontological aspects. Results were obtained by categorizing and analyzing recurring themes following a standard qualitative analysis framework.

Results: According to our study, attending a patient's funeral is acceptable for a geriatrician if a special bond has been built between the physician, the patient and/or his/her relatives. The main themes that emerged from our interviews are social acceptability, importance of medical culture and professionalism. Benevolence occurred as the main motivation for attending a patient's funeral. However, opinions varied between surveyed physicians in accordance to both personal values and systemic factors, such as the way geriatric care is organized within healthcare institutions.

Discussion: A geriatrician may be asked to partake in funerals. Considering the dearth of ethical and medical literature pertaining to this custom, a professional and personal decision is still poorly guided. This renders our research relevant for assisting a geriatrician's choice in relation to assisting funerals.

Conclusions: Our study defined the main elements influencing the presence of geriatricians at a former patient's funeral. We hope this will guide future researches.

\section{Opportunistic Biomarkers of Frailty from CT Imaging in Patients with Suspected COVID-19}

Neetika Bharaj, Pablo Solla Suárez, Jeremy Obrand, Jessica Chetrit, Fayeza Ahmad, Sarah Lantagne, David Morrison, Xiaoqing Xue, Marc Afilalo, Brent Richards, Jonathan Afilalo,

McGill University

Background: Frailty is more meaningful than chronological age for risk prediction and patient-centered decision making in COVID-19. While frailty scales may be challenging to use in this setting, computed tomography (CT) scans are often performed and provide access to opportunistic biomarkers of frailty such as skeletal muscle area (SMA).

Methods: We conducted a cohort study nested in the prospective Quebec COVID-19 Biobank. Symptomatic patients presenting to our acute care hospital were eligible if they underwent a COVID-19 test and a clinically-indicated CT scan of the chest or abdomen. Using the CoreSlicer web-based 
software, we loaded CT images and measured SMA and subcutaneous fat area at 3 pre-defined axial levels (T6, T12, L4). The current analysis focuses on scans acquired in the first 24 hours and on images at the T6 level; if not included in the scan field, multiple imputation was used based on the closest available level. The primary outcome was log-transformed hospital length of stay.

Results: Out of 770 patients included in the Biobank at our hospital, 232 met the inclusion criteria. At the T6 level in men and women, respectively, the mean SMA was 140+/-37 $\mathrm{cm} 2$ and $99+/-26 \mathrm{~cm} 2$ and the mean subcutaneous fat area was $135+/-67 \mathrm{~cm} 2$ and $217+/-133 \mathrm{~cm} 2$. SMA was inversely correlated with Rockwood's Clinical Frailty Scale (Spearman $\mathrm{R}-0.43, \mathrm{P}<0.001)$. In the linear regression model adjusted for age, sex, Charlson comorbidity index, and COVID-19 status, lower SMA was independently associated with prolonged length of stay (Beta $-0.018,95 \% \mathrm{CI}-0.001$ to -0.035 ).

Discussion: CT-based measures of muscle and fat area were reliably feasible in $97 \%$ of scans.

Conclusions: Patients with suspected COVID-19 and low muscle mass on clinical CT scans had higher risks of adverse in-hospital outcomes.

\section{Physical exercise with cognitive training and vitamin D supplementation improves brain connectivity in older adults with mild cognitive impairment (MCI). Results from the SYNERGIC Trial.}

Nick W Bray ${ }^{1}$, Suzanne T Witt ${ }^{1}$, Robert Bartha ${ }^{1}$, Timothy J Doherty ${ }^{1}$, Lindsay S Nagamatsu ${ }^{1}$, Quincy J Almeida ${ }^{2}$, Louis Bherer ${ }^{3}$, Teresa Liu-Ambrose ${ }^{4}$, Laura Middleton ${ }^{5}$, Manuel Montero-Odasso ${ }^{1}$

${ }^{1}$ Western University, ${ }^{2}$ Wilfrid Laurier University, ${ }^{3}$ Université de Montréal, ${ }^{4}$ University of British Columbia, ${ }^{5}$ University of Waterloo

Background: Physical exercise, cognitive training, and vitamin D supplementation are emerging interventions for improving brain health. How they can combine to improve brain network connectivity is still unknown.

Methods: The SYNERGIC trial is a multi-site, double-blind, five-arm, controlled trial that assesses the potential synergistic effect of combining bi-modal exercise (progressive aerobic + resistance training $=\mathrm{E}$ ), cognitive training $(\mathrm{CT})$ and, vitamin D supplementation ( $\mathrm{D}=10,000 \mathrm{IU} / 3 \mathrm{xweekly}$ ) in elderly with MCI. Interventions were delivered for 20 weeksinthefive-arm s,asfollows:Arm1 receivedthethreeactiveinterventions $(\mathrm{E}+\mathrm{CT}+$ D);Arm2receivedE $+\mathrm{CT}+$ placebo D; Arm 3 received $\mathrm{E}+$ sham $\mathrm{CT}+\mathrm{D}$; Arm 4 received $\mathrm{E}+$ sham $\mathrm{CT}+$ placebo D; and Arm 5 received all controlinterventions(controlE,shamCT,andpla ceboD).Thisanalysisincluded120participantsthatunderwent

3-Tesla resting-state fMRI pre- and post-intervention. fMRI data were analyzed using CONN.
Results: The combined intervention (arm 1) showed increased connectivity between the Fronto-parietal and Dorsal Attention $(\mathrm{p}<0.01)$ networks, as well as the Fronto-parietal and Sensorimotor networks $(\mathrm{p}<0.001)$, when compared with the full control (arm 5). These associations were also aligned with improvements seen in arm 1 vs arm 5 in a global cognition composite score (ADAS-Cog-13, $\mathrm{p}=0.01 ; \mathrm{R} 2=0.18$ ). Participants not receiving the three combined interventions did not show significant changes in brain connectivity.

Discussion: The combination of individualised and progressive exercises with cognitive training and vitamin D supplementation demonstrated increased inter-network connectivity in key brain areas related to cognition. Increased brain connectivity may explain the improved global cognitive performance seen in MCI participants receiving the three interventions.

Conclusions: Combining exercise, cognitive training, and vitamin D supplementation can increase brain network connectivity in older adults with MCI.

\section{Describing Healthcare Utilization for Men and Women Living with Dementia in Quebec}

Tammy Bui ${ }^{1}$, Claire Godard-Sebillotte ${ }^{1}$, Genevieve Arsenault-Lapierre ${ }^{1}$, Victoria Massamba ${ }^{2}$, Louis Rochette ${ }^{2}$, Isabelle Vedel ${ }^{1}$

${ }^{1}$ Mcgill University, ${ }^{2}$ Institut national de santé publique $d u$ Québec

Background: As the Canadian population ages, challenges emerge with the healthcare utilization of services for persons living with dementia (PWD). There is evidence that points to how sex/gender differences influence the healthcare utilization where older adult women may differ compared to older men when seeking and receiving treatment. The aim of this study is to describe the sex-based inequities on the healthcare utilization among PWD.

Methods: We used a repeated annual cross-sectional cohort design from 2000-2001 to 2016-2017. Data was obtained from linked population-based administrative databases from the Institut national de santé publique du Québec.

Community-dwelling older adults age $65+$ with incident cases of dementia occurring between April 1st and March 31st for each year between were included. Healthcare utilization indicators were measured as age-standardized rates. To ascertain the differences between males and females, indicators were stratified by sex over 17 cohort- years and graphed. A visual and graphical analysis allowed for clinically meaningful interpretation.

Results: A total of 237,259 persons with dementia was included in our study ( $62.7 \%$ women). Men had higher rate and number emergency department visits, rate of hospitalizations, rate of admission to long term care, rate of mortality. While women had higher prescription of cholinesterase inhibitors, benzodiazepines, and antidepressants compared to men. There 
was no meaningful difference for antipsychotic prescriptions and ambulatory visits to cognition specialists.

Discussion: These results are consistent with an Ontario study conducted by Sourial et. al.(2020).

Conclusions: Addressing the sex-based inequities in healthcare utilization in PWD will inform decision-makers for the implementation of adequate policies to promote equity in dementia care.

\section{The GeroCast Project: Delivering Living Cases in Geriatric Education through Podcasts}

Julie Cameron, Klodiana Kolomitro, Nancy Dalgarno, Jennifer Turnnidge, Lucie Pelland, Mohammad Auais Queen's University

Background: Medical and healthcare students are less interested in working with seniors and may be under-prepared to deliver services that meet the needs of this population. A novel approach that may improve students' attitudes towards working with seniors is the presentation of living case studies through podcasting technology. This study describes the development process of GeroCast; an educational, case-based geriatric podcast series created for physiotherapy students.

Methods: The creation process included six steps - infrastructure development; defining the scope of each episode; identifying/adapting existing podcast quality indicators; participant recruitment; developing and professionally editing the podcast; and quality assurance testing.

Results: From November 2019-January 2021, a podcast series of sixteen case-based episodes was created and evaluated for quality assurance (Mean duration=17.6 minutes). Each episode included background information on a health- related topic, a conversation with a senior participant sharing their related lived and living experience on the topic, reflection questions for students throughout the episode and a relevant assignment at the end of each episode.

Each episode was reviewed by members of the research team and a panel of practicing healthcare providers, then refined based on their feedback. Episodes were uploaded to Queen's University's secure streaming platform and shared with students.

Discussion: To our knowledge, GeroCast is the first educational, living case-based podcast series that also has been designed with relevant quality indicators in mind. While listening to GeroCast, students highlighted the personal connection they felt towards the seniors, and articulated key learnings. Besides physiotherapy students, living case podcasts may be an effective way to deliver geriatrics education to other healthcare students.

Conclusions: Podcasts allow for the dissemination of seniors' lived experiences, and have the potential to enhance the quality of healthcare education.

\section{Ending PJ Paralysis: A Quality Improvement Initiative.}

Frances Carr ${ }^{1}$, Angela Morgan², Shehani Devapura², Christina Williams ${ }^{2}$, Jennifer Symon ${ }^{2}$, Carolyn HoweRiddell $^{2}$, Angela Miller ${ }^{2}$, Jennifer Boone ${ }^{2}$, Kevin Ebbage ${ }^{2}$, Shalmin Jadaviji ${ }^{2}$, Pamela Mathura ${ }^{1}$

${ }^{1}$ University of Alberta, ${ }^{2}$ Alberta Health Services

Background: PJ paralysis refers to the negative effects seen amongst hospitalized patients who remain inactive and within their pajamas in hospital. Despite the many complications associated with PJ paralysis, it is a common problem within acute care facilities, which needs addressing.

Methods: A 3 month quality improvement study was conducted at the University of Alberta Hospital. The aim was for $50 \%$ of patients on ward $5 \mathrm{G} 2$ to get dressed in their own clothing by midday, sat up for all meals, and mobilizing to activities. Our intervention involved providing education and assistance to get patients dressed in their own clothing by midday, up for meals and mobilizing to activities. Measures included: daily percentage of patients dressed in their own clothing and up for all meals, weekly mobilization rates, number of nursing staff required, nursing assessment time, and complication rates (e.g. falls).

Results: 70 patients were included in the study. $14.3 \%$ of patients were dressed in their own clothing daily, and $6.4 \%$ of patients were up for all three meals. The average number of patients mobilized weekly was 4.7. A trend was observed towards decreased falls, with little change observed in staff numbers, nursing assessment time or other complication rates. Patient feedback revealed improvement in their self-identity.

Discussion: While our aim was not met, our intervention revealed improvement in patients' self-identity, consistent mobilization rates, a trend towards decreased falls, with little change observed in complications, number of staff or care assessment duration.

Conclusions: Our simple intervention had a positive impact in several areas, with few complications. Post study, this intervention continued, providing evidence of its sustainability and ease of implementation by frontline staff. Future plans include repeating a second cycle next year to determine sustainability and impact.

\section{Decision-Making Capacity Assessment (DMCA) Toolkit}

Lesley Charles ${ }^{1}$, Jasneet Parmar ${ }^{1}$, Brémault-Phillips Suzette $^{2}$, Ashley Pike ${ }^{2}$, Bonnie Dobbs ${ }^{1}$, Peter George Tian ${ }^{1}$

${ }^{I}$ Division of Care of the Elderly, Department of

Family Medicine, University of Alberta, ${ }^{2}$ Department of Occupational Therapy, Faculty of Rehabilitation, University of Alberta

Background: With an increasing elderly population, the number of persons with dementia is expected to increase. 
Consequently, the number of persons needing decisionmaking capacity assessments (DMCA) will increase. Many health care professionals do not feel ready to provide DMCA. Since 2006 we have been training allied HCPs and since 2013, we have been training physicians on DMCA. We put together a toolkit to support the DMCA Model.

Methods: A DMCA Model was developed in Alberta and aligned with provincial legislation based on literature review, surveys and interviews. It outlines DMCA best practices and includes a care map, worksheets, staff training workshops, physician training workshops, and grand rounds/Educational sessions. It has been used by physicians and other health care professionals across the care continuum. Over 1000 healthcare professionals have been trained using the DMCA Model over 12 years. The Model is supported by a 4-part toolkit.

Results: The DMCA Toolkit is a four-part practical, evidenceinformed resource consisting of the following: Part 1-The DMCA Model; Part 2-DMCA Model Implementation Framework; Part 3-Resources for Healthcare Providers (HCPs); and Part 4-Resources for Family Caregivers. Resources for HCPs are summarized from the Office of the Public Guardian and Trustee, Alberta Health Services and Covenant Health. Resources for caregivers include information on legislation, videos on the Personal Directives, financial planning and the Adult Guardianship and Trusteeship Act.

Discussion: The DMCA Toolkit supports the DMCA Model by providing details for implementation, and summarizing key resources for both HCPs and caregivers.

Conclusions: The DMCA toolkit is a culmination of 12 years of work around the DMCA Model and expands the scope of the DMCA Model providing invaluable resources.

\section{Prediction of Rehabilitation Needs After Aortic Valve Replacement}

Jessica Chetrit, Nicolo Piazza, Michael Goldfarb, Giuseppe Martucci, Lawrence Rudski, Marco Spaziano, Jean- Francois Morin, Kevin Lachapelle, Yves Langlois, Jonathan Afilalo

Mcgill University

Background: Older adults undergoing TAVR or SAVR often cannot be discharged home because they are deconditioned and require rehabilitation. Clinical and geriatric predictors of post-procedural rehabilitation needs could be addressed to anticipate and prevent this disposition but they have yet to be examined in a multicenter study.

Methods: Post hoc analysis of a prospective cohort study from 14 hospitals in 3 countries. Patients $\geq 70$ years of age were included if they underwent TAVR or SAVR and were discharged alive from hospital.

The predictor variables were: age, sex, BMI, comorbidities, frailty (measured by the EFT), disability, depression, NYHA class, LVEF, mortality, procedure type and complications.
The outcome variable was discharge to a rehabilitation facility following hospitalization. Multivariable logistic regression and Bayesian model averaging were used to select a model for future predictions.

Results: The cohort consisted of 1102 patients with a mean age of 81 . There were 646 TAVR and 374 SAVR, of which 136 and 78 were discharged to a rehabilitation facility. The final predictive model and score consisted of: advanced age, sex, high NYHA class, high EFT score, post-procedural complication, and undergoing a SAVR as opposed to TAVR.

The predicted risk of being discharged to a rehabilitation facility was $6 \%, 12 \%, 26 \%, 37 \%$, or $51 \%$ if the rehab score was $0-1,2,3,4$, or 5-6 points, respectively.

Discussion: As the rehab score increases, there is higher risk of being discharged for rehabilitation. Identifying the major predictive factors of rehabilitation needs after TAVR or SAVR and using a risk model to predict rehab needs could be used to plan patients post-procedural care.

Conclusions: A risk model for prediction of rehabilitation needs after TAVR and SAVR has been developed and could be used to proactively select patients that may benefit from prehabilitation.

\section{Usability and Impact of an Online Psychoeducation Program for Normal Age-Related Memory Changes Among Older Adults}

Danielle D'Amico' ${ }^{1}$, Iris Yusupov², Lynn Zhu³, Jordan W. Lass $^{4}$, Brian Levine ${ }^{3}$, Susan Vandermorris ${ }^{5}$, Angela

K. Troyer ${ }^{5}$

${ }^{1}$ Department of Psychology, Ryerson University, ${ }^{2}$ Department of Psychology, York University, ${ }^{3}$ Rotman Research Institute, Baycrest, ${ }^{4}$ Kunin-Lunenfeld Centre for Applied Research and Evaluation (KL-CARE), Baycrest, ${ }^{5}$ Neuropsychology and Cognitive Health Program, Baycrest

Background: Concerns about memory changes are common among older adults. The Memory and Aging Program ${ }^{\circledR}$ is a psychoeducation intervention that provides information about age-related memory changes and training in the use of memory- and lifestyle-based strategies to support brain health. An online version of the program was recently developed to increase accessibility to program participation. The objective of the current study was to assess user experience feedback among older adults who participated in the program.

Methods: Upon completion of the program, participants were directed to a feedback survey inquiring about satisfaction with the program content, memory functioning and confidence in memory abilities, and the use of memory- and lifestyle-based strategies.

Results: Among the 134 participants who enrolled, 103 individuals completed the program and provided feedback. All participants reported improvements in memory functioning, and $98 \%$ of individuals reported higher confidence in their 
memory abilities. All participants were satisfied with the pace and clarity of the learning modules, and $92 \%$ of individuals were satisfied with the organization and navigation of the interface. Participants also reported increases in physical activity (72\%), use of stress-reduction techniques (72\%), cognitive engagement (68\%), healthy diet (58\%), social engagement $(48 \%)$, and the use of memory strategies (63-97\%).

Discussion: Results show high satisfaction with the program among participants, and a positive impact on self-reported uptake of memory- and lifestyle-based strategies. Findings have implications for the effective use of online tools to support brain health in later life.

Conclusions: The online Memory and Aging Program $₫$ may be a promising avenue to promote brain health among the growing aging population, especially in the context of the shift to virtual care during the COVID-19 pandemic.

\section{Co-Designing Patient Safety Conversations in Long Term Care}

Vivian Ewa, Jennifer Lee, Chris Jones, Abram Gutscher Alberta Health Services

Background: At Alberta Health Services (AHS), current patient safety strategy is reactive in long term care (LTC). Recognizing the limits of this strategy, our goal is to move to a proactive patient safety approach. Research in patient safety culture indicates that conversations on safety is critical for proactive strategy (Vincent, et al, 2013). Our project aims to co- design tools to enhance patient safety conversations between staff, families and residents in LTC using design thinking methodology.

Methods: We interviewed forty individuals at two LTC sites to understand what families, staff and residents think about safety. Teams from LTC participated in a design sprint to address our challenge, "How might we support meaningful safety conversations between residents, families and staff?" Data and themes from interviews informed the co-design. Two prototypes were created from the sprint and tested in LTC environments.

Results: The first prototype, "Me and My Needs" is a visual representation of key information important to the resident. This prototype encourages rich conversation between residents and staff, and evolves as the resident's needs change. The second prototype, "Conversation Cards" is a pocket sized card with color coded safety questions (based on level of rapport) for staff and families to discuss with each other.

Discussion: Both prototypes facilitated relationship buildingcritical for safety conversations. Moreover, organizational factors (role modelling conversations) encourages engagement in conversation.

Conclusions: This project achieved co-design intent through strong partnerships with our key stakeholders. The resident and family voice was represented at each stage, and determined the final prototype. Moreover, the project identified organizational factors critical to support safety conversations. Finally, both prototypes will be implemented at participating sites, and the project team is sharing the prototypes with other LTC sites for widespread adoption.

\section{Reporting exercise principles in exercise intervention programs for community- dwelling older adults with fear of falling: A systematic review}

Chengying Feng ${ }^{1}$, Tony Adebero ${ }^{2}$, Vincent DePaul ${ }^{1}$, Afshin Vafaei $^{3}$, Kathleen Norman ${ }^{1}$, Mohammad Auais ${ }^{1}$ ${ }^{1}$ Queen's University, ${ }^{2}$ University of Western Ontario, ${ }^{3}$ Lakehead University

Background: Fear of Falling (FOF) contributes to activity restriction and institutionalization among older adults; exercise interventions have been linked to reduction in FOF. Adhering to exercise principles (specificity, progression, overload, reversibility, diminished return, and initial value) and adapting optimal exercise parameters (frequency, intensity, time and type of exercise [FITT]) are fundamental to optimizing the effectiveness of exercise interventions. Failure to provide these details of exercise interventions in the literature is a serious barrier to implementation in practice. The purpose of this systematic review was to describe FOF exercise interventions in older adults and evaluate the extent to which these interventions followed the exercise principles and reported FITT components.

Methods: Randomized Controlled Trials (RCTs) of FOF exercise interventions in older adults ( $\geq 65$ years) were identified from MEDLINE, EMBASE, PsycINFO, and CINAHL databases. The methodological quality of RCTs was assessed using the PEDro scale.

Results: Seventy-five RCTs were included in our review after screening 511 and reviewing 158 articles. Regarding exercise principles, specificity was reported in $92 \%$ of trials, progression in $72 \%$, reversibility in $32 \%$, overload in $31 \%$, diminished return in $21 \%$, and initial value in $8 \%$. For exercise parameters, $97 \%$ RCTs reported exercise type, $89 \%$ frequency, $85 \%$ time, and $25 \%$ intensity.

Discussion: Future research involving exercise interventions should use a valid tool such as the Consensus on Exercise Reporting Template to report the essential components of exercise interventions.

Conclusions: Most exercise principles and intensity from FITT components were not adequately reported in included RCTs. These shortcomings undermine efforts to fully examine factors contribute to the effectiveness of FOF exercise interventions. To facilitate evidence-based practice, more attention must be given to designing and reporting components of therapeutic exercise programs. 


\section{Prevalence of Frailty Among Hospitalized Older Adults in New Brunswick, Canada: An Administrative Data Based Study}

Molly Gallibois ${ }^{1}$, Kyle Rogers ${ }^{2}$, Chris Folkins ${ }^{2}$, Pamela Jarrett $^{3}$, Sandra Magalhaes ${ }^{2}$

${ }^{1}$ University of New Brunswick, ${ }^{2}$ Institute for Research, Data and Training, University of New Brunswick, ${ }^{3}$ Horizon Health Network, Department of Geriatrics Saint John and Faculty of Medicine, Dalhousie University

Background: Frailty is a strong predictor of adverse health outcomes including falls, hospitalization, and nursing home admission. Characterizing the prevalence of frailty within a population can help guide policy development and predict health service resource needs. Here we describe the prevalence of frailty and characterize the frail population among hospitalized older adults in New Brunswick (NB), Canada, which to our knowledge has not previously been reported.

Methods: Adults over age 65 were identified using the Citizen Database, which contains demographic information of NB residents with a valid Medicare number. Of this group, individuals hospitalized between April 1, 2017 and March 13, 2019 were identified using information from Discharge Abstract Data (DAD) records. Frailty was assessed using the Hospital Frailty Risk Score (HFRS), a validated frailty index derived from patient information in DAD records.

Individuals with a HFRS score ranked as intermediate or high were considered to be frail.

Results: Among 150,000 older adults, 36\% ( $=55,675 ; 26,800$ males) were hospitalized during the two-year study period. The overall prevalence of frailty was $20.9 \%$, with slightly lower prevalence among males $(19.2 \%)$ than females $(22.6 \%)$. Prevalence increased with age: $12.5 \%$ in the $65-74$ age group, $24.3 \%$ in the $75-84$ age group and $41.1 \%$ for those over age 85 . In the youngest age group frailty was slightly more prevalent in males, while in the older two age groups frailty was more common in females.

Discussion: The distribution of frailty in NB is in line with that reported in other jurisdictions. Our future work will investigate the association between frailty and health care service utilization, such as nursing home admissions.

Conclusions: We demonstrate the feasibility of the HFRS to identify and characterize frailty using administrative data in Canada.

\section{Applying Patient Oriented Research in the Context of Long-term Care: Development of a Diverse Stakeholder Knowledge Translation Network}

Kirstian Gibson ${ }^{1}$, Yuchen Gao ${ }^{1}$, Katherine M. Ottley ${ }^{1}$, Thomas Qiao' ${ }^{1}$, Ivy Myge ${ }^{1}$, Roslyn M. Compton ${ }^{1}$, Abigail Wickson-Griffiths ${ }^{2}$, Paulette V. Hunter ${ }^{3}$, Saskatchewan Long-Term Care Network
${ }^{1}$ University of Saskatchewan, ${ }^{2}$ University of Regina, ${ }^{3}$ St. Thomas More College

Background: The emergence of the COVID-19 pandemic immediately created several challenges for the long-term care (LTC) system. In response, the Saskatchewan Long-Term Care (SK LTC) Network formed in March 2020.

Methods: The network includes a diverse group of LTC stakeholders, including interprofessional health providers, researchers, family caregivers, and trainees, who gathered with the purpose of serving as a resource to the LTC sector in Saskatchewan during and ideally beyond the pandemic.

Results: The network is successfully establishing itself within Saskatchewan as an evolving knowledge translation hub that emphasizes relational strategies and a patient-oriented approach. Patient-oriented approaches engage patients, families, and/or caregivers in the construction and dissemination of knowledge to ensure that the evidence used to guide clinical practice reflects patient priorities and leads to better health outcomes.

Discussion: In this presentation, we will review the steps taken to develop a patient-oriented network to address the needs of family caregivers and residents of LTC. We will also describe our patient-oriented approach to developing a mandate and guiding principles, and our current and past activities. Finally, we will explore the potential role for patient-oriented research and knowledge translation networks in addressing older adult and LTC issues in Canada.

Conclusions: Through this presentation, participants will learn about one approach to engaging a patient-oriented knowledge translation network focused on the needs of the LTC sector.

\section{Health-seeking behavior related to selected dimensions of wellness in community-dwelling older adults}

Navjot Gill ${ }^{1}$, Denise Connelly ${ }^{2}$

${ }^{1}$ University of Waterloo, ${ }^{2}$ Western University

Background: Older people generally prefer to 'stay-put' in their own homes. Informed by the Seven Dimensions of Wellness, a component of the International Council on Active Ageing, measures of physical function (i.e., physical), fall risk (i.e., environmental), and psychosocial factors (i.e., emotional, spiritual and social) related to wellbeing were selected, including awareness of community support services. It is assumed that they intersect to influence the health-seeking behaviour of older adults.

Methods: The objectives of this cross-sectional design study were to 1) investigate the relationship among dimensions of wellbeing with health-seeking behaviour in older adults and 2) to determine if there was a difference(s) in health- seeking behaviour between men and women or between age groups, 
i.e., less than 75 years old, and 75 years and older. A sample of 99 older adults living at home in London, ON, aged $\geq 65$ years, ambulatory (with/without gait aid) and without executive function impairment were recruited. Validated outcome measures were administered. Data were analyzed using multiple linear regression and Mann-Whitney U-Test.

Results: Health-seeking behaviour had a significant positive relationship with the awareness of community support services. However, no significant relationship was found between health-seeking behaviour and dimensions of wellbeing. Nodifferenceinhealth-seekingbehaviourwasfoundbetweenmenandwomenorbetweenagegroups.

Discussion: Awareness of community services may be an essential strategy for clinicians to provide older adults with the information they need to make decisions regarding their health and promote health-seeking behaviour.

Conclusions: More research is required to understand healthseeking behaviour and factors from other physical and cognitive models to clarify better the factors related to this concept. It is crucial to comprehend health-seeking behaviour as it may be the driving force behind assisting ageing-in-place.

\section{Care and health services use in community- dwelling persons with dementia: is Quebec achieving equity?}

Claire Godard-Sebillotte ${ }^{1}$, Genevieve Arsenault-Lapierre ${ }^{1}$, Tammy Bui ${ }^{1}$, Nadia Sourial ${ }^{2}$, Amélie Quesnel-Vallée ${ }^{1}$, Victoria Massamba ${ }^{3}$, Louis Rochette ${ }^{3}$, Isabelle Vedel ${ }^{1}$ ${ }^{1}$ Mc Gill University, ${ }^{2}$ Université de Montréal, ${ }^{3}$ Institut national de santé publique du Québec

Background: Evidence shows inequities associated with socioeconomic status (SES) in the care of persons with dementia. However, evidence is scarce and conflicting. There is an urgent need to describe the association between socioeconomic status, care received, and health services use in persons with dementia in order to ensure equity across all levels of SES. We described prevalence and trends of care and health service use across SES in community-dwelling persons with dementia in Quebec.

Methods: We conducted a repeated yearly cohort study of community-dwelling persons with incident dementia using the Quebec health administrative database (2000-2017). We described age standardized rates per 100 person-year of indicators of care and health service use during the year following diagnosis across 5 SES levels based on the area of residence. We used the material deprivation Pampalon index: a composite measure of the level of education, employment, and income of the census dissemination area level. We assessed the differences across SES groups by graphical assessment and expert consensus.

Results: Among the 237,635 community-dwelling persons with a new diagnosis of dementia between 2000 and 2017
(63\% women, mean age 81,3 year), those living in the most deprived areas compared to those living in the least deprived areas had more hospitalization (44,15 vs 35,67 per 100 person year), more ED visits $(63,09$ vs 53,84$)$. In addition, theywereprescribedmorepotentiallyinappropriatemedicationsincluding antipsychotic( 33,55 vs 26,60$)$ and benzodiazepines $(42,73$ vs $34,52)$. In contrast, prescription of anti-dementia treatment, visits to primary care physician, and mortality were comparable in every socio-economicgroup.

Discussion: Our results are consistent with the only other Canadian study on this topic. This British Columbia study showed associations between low income and less counselling, referrals, and more antipsychotics drugs.

Conclusions: These socio-economic differences may signal inequities in the care received in persons with dementia. Future research should investigate these associations to better understand their underlying causes and the best way to tackle them, in order to offer equitable care to the entire Canadian population living with dementia.

\section{Opioid-Induced Neurotoxicity As The Culprit For Recurrent Falls: A Case Report}

Bradley Godwin ${ }^{1}$, Ripa Akter ${ }^{2}$

${ }^{1}$ University of Ottawa, ${ }^{2}$ The Ottawa Hospital, Division of

Geriatric Medicine

Background: Chronic pain and opioid use are extremely common in older adults. In Canada, those over 65 have consistently received more new opioid prescriptions and have a higher proportion that go on to long-term opioid therapy (24.8\%) than any other age group (CIHI, 2019). Opioid-related adverse effects include constipation, nausea, dizziness, sedation, respiratory depression, and falls. Older adults are at greater risk for adverse effects including toxicity due to drug-drug interactions, multimorbidity, and age-related physiologic changes.

Methods: A 66-year-old female admitted with decompensated congestive heart failure was referred to Geriatric Medicine for evaluation of recurrent falls and cognition. She complained of involuntary muscle twitching in all extremities, which resulted in falls during her admission. She was receiving morphine $30 \mathrm{mg}$ twice daily for chronic pain.

Physical examination revealed marked inattention with positive and negative myoclonus in all extremities without focal neurologic deficits. Laboratory investigations revealed acute renal impairment.

Results: She was subsequently rotated from morphine to hydromorphone and diuretics were decreased. Collateral history revealed similar isolated episodes in the preceding year leading to falls-related admissions. During inpatient stay, myoclonicjerksandmobilitysubstantiallyimprovedfollowingopioidrotation.Bothrenalimpairmentanddelirium resolved.

Discussion: A diagnosis of opioid-induced neurotoxicity (OIN) was made based on myoclonus and delirium in the 
context of chronic opioid use after other causes including primary neurological conditions were excluded. OIN can also present with cognitive dysfunction, hallucinations, allodynia, hyperalgesia, and seizures. Current evidence for opioid-associated neurotoxic effects in patients with renal impairment consists of very low-quality studies. One prospective study found that $26 \%$ of 109 patients receiving morphine for chronic-malignancy related pain developed myoclonus.

Conclusions: The patient was discharged on oral hydromorphone with complete resolution of myoclonus and improved cognition.

\section{Using a social ecological approach to understand transitions from hospital to home for frail older adults.}

Natasha Hanson ${ }^{1}$, Emily Kervin ${ }^{1}$, Leanne Skerry ${ }^{1}$, Pamela Jarrett ${ }^{2}$, Rose McCloskey ${ }^{3}$

${ }^{1}$ Horizon Health Network, ${ }^{2}$ Dalhousie University; Horizon Health Network, ${ }^{3}$ University of New Brunswick, Saint John

Background: The social ecological model (SEM) is used as a theoretical framework within which hospital-to-home transitions for frail older adults can be understood. The SEM allows for the exploration of the dynamic interrelationships of patients with the various spheres of influence that shape how transitions in care are experienced.

Methods: A secondary social ecological analysis was conducted on data collected from a larger study. The original study consisted of an exploration of the transition process for frail older adults who were discharged from hospital based geriatric units to their home in the community in New Brunswick, from the perspectives of patients, family caregivers, and healthcare providers. The study was prospective multi-phased, mixed methods in design, with purposive sampling and cross-case thematic analysis of Phase 2, triangulated with Phase 1 findings. Phase 3 consisted of a review of relevant policies at the local, provincial, and national levels.

Results: Interpersonal factors impacting transitions included: characteristics of patients and family caregivers, such as health concerns, education/knowledge, and income. Interpersonal factors included: communication with healthcare providers, discharge instructions received, and adherence to recommendations. Institutional factors also played a role, such as: absence of formalized institutional policies, lack of communication and collaboration between hospital and community services, and lack of continuity of care. Community level factors included: variability of timely available community services, affordability of community services, and medical follow-up. The lack of formalized public policy within New Brunswick was also indicated as a factor.

Discussion: Multiple facilitators and modifiable barriers were identified by patients, family caregivers, healthcare providers, as well as policy review, across all levels of the model.
Conclusions: In using a social ecological approach this study demonstrates the complexity of the hospital-to-home transition for frail older adults.

\section{Geographic Variation in Bone Mineral Density and Prevalent Fractures in Canada}

Nazila Hassanabadi ${ }^{1}$, Claudie Berger ${ }^{2}$, Alexandra Papaioannou $^{3}$, Angela M Cheung ${ }^{4}$, Elham Rahme ${ }^{5}$, William D Leslie ${ }^{6}$, David Goltzman ${ }^{5}$, Suzanne Morin ${ }^{5}$

${ }^{1}$ Mc Gill University, ${ }^{2}$ Research Institute of the McGill University Health Centre, ${ }^{3}$ Department of Medicine, McMaster University, ${ }^{4}$ DepartmentofMedicine, UniversityofT oronto, ${ }^{5}$ DepartmentofMedicine,McGillUniversity/Research InstituteoftheMcGillUniversityHealthCentre, ${ }^{6}$ Departmentof Medicine, UniversityofManitoba

Background: Purpose:Theprevalenceofosteoporosisandfra cturesdiffersacrosscountriesandregions. Weaimedtodescribe sex-specific total hip bone mineral density (Hip-BMD) and prevalent major osteoporotic fractures (MOF) variation between Canadianprovinces.

Methods: Methods:Weusedbaselinedataof27,244Canadians $(13,828$ women, $13,416 \mathrm{men})$ aged50+yearsparticipatingin the Canadian Longitudinal Study of Aging (2012-2015). Linear and logistic regression models were used to examine associations between the province of residence and Hip-BMD and self-reported MOF, stratified by sex and adjusted for important covariates (Ontario [ON]:reference).

Results: Results: The mean (SD) age of participants was 64.4(9.4) years in women and 64.8(9.4) in men. Mean BMI $(\mathrm{kg} / \mathrm{m} 2)$ waslowestinBritish-Columbia(BC;27.4[5.1])andhi ghestinManitoba(MB;29.0[6.1]). Themean10-yearMOFrisk (FRAX ${ }^{\circledR}$ ) was not different between provinces in both women and men. Women and men from $\mathrm{BC}$ had the lowest mean Hip-BMD and prevalent MOF. Alberta (AB) (12.3\%) had the highest proportion of participants reporting recent falls, while MB $(8.6 \%)$ had the fewest. Linear regression demonstrated significant differences in Hip-BMD; women and men from $\mathrm{BC}$ and $\mathrm{AB}$, and women from $\mathrm{MB}$ and Nova-Scotia (NS) had lower, while men from NS had higher adjusted BMD than ON. Adjusted Odds Ratios (95\% confidence intervals) for prevalent MOF were significantly lower in $\mathrm{BC}$ women ( 0.57 ; $0.41-0.81)$ and men $(0.32 ; 0.19-0.53)$, and NS men $(0.49 ; 0.26-$ $0.92)$, while it was higher in women from NS $(1.48 ; 1.04-2.10)$ compared to $\mathrm{ON}$. Results were similar when restricting the analyses to participants of Whiteancestry.

Discussion: Discussion: Geographical variations in Hip-BMD and prevalent MOF between provinces persisted after adjusting for important covariates.

Conclusions: Conclusion: These observed variations could be related to unmeasured individual, social and environmental factors. 


\section{Access to Community-Based Specialized Geriatric Care in Ontario: Proposing a Decision-Support Tool to Facilitate the Referral Process}

Sophie Hogeveen ${ }^{1}$, George Heckman ${ }^{2}$, Kelly Milne ${ }^{3}$, John Hirdes ${ }^{2}$

${ }^{1}$ McMaster University, ${ }^{2}$ University of Waterloo, ${ }^{3}$ Regional Geriatric Programs of Ontario

Background: The role played by geriatric medicine (GM) in caring for seniors in the community with complex needs, such as those receiving publicly-funded home care (HC) services, has not been well described in Ontario. Study objectives were to explore service use patterns, outcomes, and characteristics associated with GM contact, and to propose a mechanism for more equitable referral and access to care.

Methods: This was a population-based retrospective cohort study of older HC clients in Ontario $(\mathrm{N}=196,444 ; 2012$ 2015). Standardized HC assessments were linked to health administrative datasets (out-patient physician and acute care services use). Descriptive statistics were used to describe service use patterns. Multivariable generalized estimating equation modeling was used to examine associations between client characteristics and outpatient GM contact in 90 days post-assessment. Based on results, provincial stakeholders created a standardized decision- support tool to identify $\mathrm{HC}$ clients who would most benefit from GM referral.

Results: $49.6 \%$ of older $\mathrm{HC}$ clients had $\geq 4$ physician contacts post-assessment; only $5.2 \%$ had GM contact. In the final multivariable model (region-adjusted), female sex, difficulties accessing home, impaired locomotion, recovery potential, hemiplegia/hemiparesis, and cancer were associated with lower odds of GM contact. Age, worsened decision-making, dementia, hallucinations, Parkinsonism, osteoporosis, and caregiver distress/institutionalization risk were associated with higher odds of contact. A tool was proposed that identifies $\mathrm{HC}$ clients at risk for caregiver distress/institutionalization (based on current practice), with medical instability and complexity (based on provincial expertise), and needs within the Geriatric 5Ms for GM referral.

Discussion: Few older HC clients were in contact with community-based GM, despite having complex medical needs and frequent contact with the health system.

Conclusions: A decision-support tool could inform workforce planning, improve care provider collaboration, and promote more rational and equitable resource allocation.

\section{Factors Influencing Primary Care Visits by Older Adults in Nova Scotia With and Without Dementia}

Natalie Horne ${ }^{1}$, Emily Marshall ${ }^{2}$, Melissa Andrew ${ }^{3}$ ${ }^{1}$ Dalhousie University, ${ }^{2}$ Dalhousie Departments of Family Medicine, Psychiatry \& Community Health and Epidemiology, ${ }^{3}$ Dalhousie University Depertment of Geriatric Medicine
Background: Age, sex, multimorbidity, and polypharmacy have been implicated in increased health care utilization These outcomes, however, have not specified the impact at the level of primary care. The objective of this study was to determine the extent to which previously identified factors affect the number of visits in primary care by older adults and explore the burden of these factors in adults with dementia.

Methods: This retrospective cohort study used de-identified electronic medical record data from clinics participating with the Maritime Family Physician Research Network, a regional network of the Canadian Primary Care Sentinel Surveillance Network (CPCSSN). 16235 subjects were included in the study, 1032 with a dementia diagnosis.

Backward linear regression analysis was used to determine the factor with the greatest influence on primary care visits.

Results: Multimorbidity was found to have the greatest influence on number of primary care visits $(B=2, p=<0.001)$. Impacts of older age and a greater number of prescriptions were also statistically significant. Patients within the dementia group had higher multimorbidity, were older, prescribed more medications, and as predicted had a higher number of primary care visits.

Discussion: The findings of this study support trends identified in previous research. The study adds that primary care is also impacted by increasing multimorbidity. Individuals with dementia carried a greater disease burden and a greater number of primary care visits versus those without dementia. The findings of this study support the growing need for resource allocation to support a multimorbidity approach in primary care.

Conclusions: Increasing multimorbidity had the greatest impact on primary care visits versus patient age, sex, and polypharmacy. Patients with dementia had a higher number of multimorbid conditions and more primary care visits.

\section{Impact of Cognitive Impairment in Older Adults on 1-year Outcomes after Elective Total Hip Arthroplasty for Osteoarthritis: Case-control study}

Susan Hunter, Tony Adebero, Lyndsay Somerville, Brent Lanting

University of Western Ontario

Background: The majority of total hip arthroplasty (THA) surgeries are performed in adults over 65 years. The aging population will see more people with cognitive impairment as candidates for and recipients of elective THA. It is unknown if people with cognitive impairment achieve the same benefits of improved pain and physical function from the surgery. The aim was to evaluate the surgical and functional outcomes in people with and without cognitive impairment after elective primary THA for osteoarthritis.

Methods: This is a case-control study. Participants were matched on age and gender (31 cognitively impaired and 31 
cognitively healthy) at one year after THA. Scores $\leq 25$ on Montreal Cognitive Assessment denoted cognitive impairment. Surgical outcomes were Harris Hip Score, Western Ontario McMaster Osteoarthritis Index, SF-12 and Falls Risk for Older People in a Community Setting (FROP-Com). Functional outcomes were Timed Up \& Go (TUG) test, Step Test, 30 -second Sit to Stand Test, single and dual-task gait testing. Independent t-tests evaluated group differences. Standardized mean differences (SMD) were calculated for effect sizes.

Results: There were no significant differences $(p>0.05)$ between groups on all surgical outcomes. The FROP-Com score demonstrated mild risk for both groups. Functional outcomes were significantly different on TUG $(p=0.029)$, Step Test $(\mathrm{p}=0.009)$ and single-task gait velocity $(\mathrm{p}=0.025)$ in favor of the cognitively healthy, all with a moderate effect size.

Discussion: Importantly, there were no differences on the surgical outcomes or fall risk. There were differences in favour of the cognitively healthy in some functional outcomes. Future research should evaluate gains from pre to post- surgery within groups. The perspectives of people with dementia and their caregivers on the experiences of the surgery and return to important life activities is needed.

Conclusions: People with mild cognitive impairment achieve similar results on surgical outcomes and present with some deficits in function.

\section{Impact of spencerTM on Medication Adherence, Usability and Caregiver Burden in Patients with Chronic Conditions in the Community Setting: A Prospective, Observational Study}

Jessica Ivo, Teresa Pitre, Sadaf Faisal, Kristen Antunes, Kasumi Oda, Tejal Patel

University of Waterloo School of Pharmacy

Background: Although the use of real-time monitoring of medication adherence is now available, there is limited knowledge of theuseofsuchtechnologyinpatients'homes.Th eaimofthisstudyistoexaminemedicationadherence,usability and impact on caregiver burden with the use of spencerTM in community dwellingadults.

Methods: This was a prospective, observational study where participants used spencerTM for their medication administration for 6 months. Real-time adherence data was collected using the spencerTM AdhereNet Platform. Adherence was defined as a dose intake within a +/-2-hour window of scheduled administration time. Usability, usefulness, satisfaction, and caregiver burden were measured using the System Usability Scale (SUS), the Usefulness, Satisfaction and Ease of Use questionnaire (USE) and Caregiver Burden Scale (CBS).

Results: Of the 49 adult participants, 55\% were female $(\mathrm{N}=27 / 49)$ with a mean age of 64.8 years $(\mathrm{SD}:+/-11.8$, Range: 44- 89). Of the 11 caregiver participants, $91 \%$ were female. The average monthly adherence over six months was
98\% (SD: +/-3.3; Range: 77.4-100). The average SUS score was 85.83 (SD: +/-12.6, Range: $48-100 ; \mathrm{N}=48$ ). Of the 47 participants who provided data, $45(96 \%)$ rated spencerTM as easy and $44(94 \%)$ as simple to use, while $44(94 \%)$ were satisfied with the product. CBS scores were $7 / 10$ (SD: +/-2.6, Range: $1-10$ ) and 3/10 (SD: +/-2.8, Range: 0-8), pre and post spencer TM use, respectively.

Discussion: Spencer TM was easy and simple to use, with a mean adherence of $98 \%$. It has the potential to improve caregiver burden.

Conclusions: Technologies such as spencerTM could be a useful solution for medication management in patients on chronic drug therapy.

\section{The association between orthostatic hypotension and gait in cognitively impaired elderly adults}

Gabriella Jacob $^{1}$, Malcolm Sherwood ${ }^{1}$, Shabhaz Malik ${ }^{2}$, Jacquie Baker ${ }^{3}$, Jaspreet Bhangu ${ }^{1}$

${ }^{1}$ Western University, ${ }^{2}$ Kansas City University, ${ }^{3}$ Libin Cardiovascular Institute of Alberta, Department of Cardiac Sciences, Cumming School of Medicine, University of Calgary

Background: Orthostatic hypotension $(\mathrm{OH})$ is defined as a decrease in blood pressure $(\geq 20 / 10 \mathrm{mmHg})$ when changing position from supine to standing. $\mathrm{OH}$ is associated with increased fall risk; however, the associations between gait and $\mathrm{OH}$ in patients with cognitive impairment (CI) remains unclear. The purpose of this study was to test the hypothesis that gait speed and dual task gait speed were slower in CI patients with concomitant $\mathrm{OH}$.

Methods: A retrospective analysis of a sample of participants from the Canadian Consortium of Neurodegeneration and Aging $(\mathrm{n}=246)$. To confirm a diagnosis of $\mathrm{OH}$, heart rate and blood pressure was measured supine and after 3 minutes of standing. Single factor ANOVA was used to compare patients with $(n=39)$ and without $\mathrm{OH}(\mathrm{n}=207)$. A secondary analysis was performed comparing those with $\mathrm{CI}+\mathrm{OH}$ to healthy controls $(\mathrm{HC})+\mathrm{OH}$.

Results: Patients with $\mathrm{OH}$ demonstrated slower mean gait speed (5.8 \pm 1 seconds) compared to patients without $\mathrm{OH}$ (5.6 \pm 1.5 seconds $; p=0.55)$.PatientswithOHalsodemonstrate dslowermeandualtaskgaitspeed $(6.9 \pm 1.7$ seconds $)$ compared to those without $(6.5 \pm 2.3$ seconds; $p=0.26)$. Fear of falling was higher in $\mathrm{OH}$ patients, but this did not meet statistical significance. There were no statistically significant differences detected between patients with $\mathrm{CI}+\mathrm{OH}$ compared toHC $+\mathrm{OH}$.

Discussion: Patients with $\mathrm{OH}$ demonstrated slower mean gait, dual task gait speed and increased fear of falling. These parameters failed to reach statistical significance; however, this may be due to small sample size.

Conclusions: $\mathrm{OH}$ remains an important clinical risk factor in individuals with cognitive impairment; however the factors 
that may pre-dispose an individual to increased fall risk remains unclear. We have demonstrated important signals that warrant further analysis with a larger cohort.

\section{Impact of a geriatric consultation service on outcomes in older trauma patients: a retrospective pre-post study.}

Lara Khoury $^{1}$, Joy Moors ${ }^{1}$, Jacynthe Lampron ${ }^{1}$, Sonshire Figueira $^{2}$, Marie-Joe Nemnom ${ }^{3}$, Debra Eagles ${ }^{1}$

${ }^{1}$ The Ottawa Hospital, ${ }^{2}$ Ottawa Regional Trauma Program, ${ }^{3}$ Clinical Epidemiology Program, Ottawa Hospital

Research Institute

Background: Early geriatric involvement is recommended for older trauma patients. We wished to determine the impact of geriatric consultation on mortality, hospital length of stay and discharge destination in older patients who were admitted to our Level 1 trauma unit.

Methods: We completed a health records review of trauma unit patients, age $\geq 75$ years old with Injury Severity Score (ISS) $\geq 12$, before $(11 / 2015$ - 10/2017) and after (11/2017 10/2019) implementation of a geriatric trauma consultation initiative. Primary outcomes were mortality, hospital length of stay, discharge destination. Secondary objectives were adherence to the geriatric trauma consult process and identification of geriatric-specific issues. A multivariable analysis controlling for age, gender, multi-morbidity and ISS was undertaken.

Results: 157 patients pre-implementation and 172 postimplementation with mean age 83.8 years old and $53.8 \%$ female were included. Geriatric consultation had no impact on in-hospital mortality (OR $0.70(95 \%$ CI $0.31-1.58)$ ) or length of stay (ß $0.68(95 \%$ CI -1.35-2.72)). Patients who received a geriatric consultation were more likely to be discharged back home (OR 2.01 (95\% CI 1.24 - 3.24). The adherence to the consultation process was $99.4 \%$.

Discussion: There was a high level of adherence to the consultation process, resulting in the identification of multiple geriatric concerns, including mobility, pain, and cognitive impairment. Our multivariable analysis that controlled for age, gender, multi-morbidity and injury severity found no impact on hospital LOS or mortality; however, patients seen by the geriatric consultation team were twice as likely to be discharged back to their home environment.

Conclusions: Older trauma patients who receive a geriatric consultation are more likely to be discharged home. Collaboration between trauma and geriatric specialists is beneficial and may lead to meaningful improvements in outcomes for older trauma patients.

\section{Development of a Geriatric Emergency Liaison Service in the Tertiary Hospital: A QI study}

Kristine Kim ${ }^{1}$, Jennifer Koop ${ }^{2}$, Lara Khoury ${ }^{1}$

${ }^{1}$ University of Ottawa, ${ }^{2}$ The Ottawa Hospital
Background: Older adults account for an increasing proportion of Emergency Department (ED) visits and up to $43 \%$ of admissions, whereafter they are vulnerable to acute functional decline and deconditioning. The objective was to develop and evaluate the integration of a dedicated geriatrician in the ED who provided early access to specialized comprehensive geriatric care. The aim was to increase ED-generated consults from a baseline of 2 to 6 per month and facilitate timely transfers to the Geriatric Medicine Unit (GMU).

Methods: This quality improvement project followed The Ottawa Hospital Innovation Framework and included collaboration with stakeholders from the ED, Geriatrics, and Medicine. Evaluation of the process for identifying patients in need ofgeriatricassessmentandtransferstoGMUwasaccomplishedusingoutcome(totalnumberofconsults), process (GMU transfers, bed availability) and balancing measures(LOS).

Results: During the first PDSA cycle from January 14 to March 13, 2020, there were 51 ED-generated consults resulting in 36 patient transfers to GMU (71\%) with 26 directly from the ED (73\%). Run Chart analysis showed evidence of special cause variation in the number of consults generated early in ED. The Average LOS was 17.4 days. The average ED LOS was 34.1 hours. The average ALC LOS was 2.9 days. The 30 -day readmission rate was $7 \%$.

Discussion: There was a significant increase in the average number of monthly ED consults from 2 to 17 . Consequently, a higher number of patients were seen earlier in their hospital course and transferred to GMU directly from the ED compared to previous years.

Conclusions: The Geriatric ED Liaison Service demonstrated a demand for early geriatric involvement which contributed to improved patient flow from the ED. Subsequent PDSA cycles will build on these findings.

\section{"Patient preference" approach to recruiting research participants: Perceptions of primary care-based memory clinic team members}

Linda Lee ${ }^{1}$, Loretta M. Hillier ${ }^{2}$, Tejal Patel ${ }^{3}$, Susie Gregg ${ }^{4}$, Kathy Hickman ${ }^{5}$, Kelly Kay ${ }^{6}$, Caitlin Agla ${ }^{7}$

${ }^{1}$ Centre for Family Medicine Family Health Team, McMaster University, ${ }^{2}$ GERAS Centre for Aging Research, ${ }^{3}$ Centre for Family Medicine Family Health Team; School of Pharmacy, University of Waterloo, ${ }^{4}$ Canadian Mental Health Association Waterloo Wellington, Guelph, ${ }^{5}$ Alzheimer Society of Ontario, ${ }^{6}$ Provincial Geriatrics Leadership Office, ${ }^{7}$ Centre for Family Medicine Family Health Team

Background: Within Multispecialty Interprofessional Team (MINT) Memory Clinics there is interest in creating a standardized approach for research participant recruitment. A "patient preference" approach to recruitment was co-designed by persons with dementia, caregivers, clinicians, and researchers. 
Interested patients complete a survey stating their preferences for research participation. Preferences are then matched to existing studies by a regional coordinator. This study describes memory clinic team members' perceptions of this approach.

Methods: Surveys were distributed to 531 participants of a MINT Memory Clinic education event. 5-point Likert-type scales were used to measure: importance of engaging patients in research; satisfaction with this approach; clinics' role in study recruitment; regional coordinator role in facilitating recruitment; willingness to adopt this approach; and, the extent to which this approach could negatively impact care or create conflict of interest.

Results: 187 surveys were completed by physicians (48), nurses (60), social workers (33), and other interprofessional team members (46). $74 \%$ perceived that it is "very" (52\%) or "extremely" (22\%) important to engage patients in research; $23 \%$ rated this as "somewhat" important. Most were "satisfied" or "very satisfied" with this recruitment approach (91\%), the limited responsibility of clinics in study recruitment (83\%), and the Regional Point Person role (89\%); fewer than $2 \%$ expressed dissatisfaction with these items. The majority of respondents indicated that this approach would have minimal negative impact ("not at all" or "a little bit") on patient care $(85 \%)$ or create conflict of interest $(82 \%) .67 \%$ were "very" or "extremely" willing to adopt this approach.

Discussion: These findings are suggestive of MINT memory clinics' support for this recruitment approach.

Conclusions: Further research is needed to gather the perspectives of researchers and determine the feasibility of implementing this approach.

\section{COVID-19 Pandemic Outreach to Frail Older Adults in Primary Care}

Linda Lee ${ }^{1}$, Loretta M. Hillier ${ }^{2}$, Tejal Patel ${ }^{3}$, Jillian Carducci $^{3}$, Kara Skimson 4 , Sharon Dillon-Martin ${ }^{4}$, Lissa Kuzych ${ }^{4}$, Lindsay Beuermann ${ }^{4}$, Ruchi Parikh ${ }^{4}$, Catherine Lee ${ }^{4}$

${ }^{I}$ Centre for Family Medicine Family Health Team; McMaster University, ${ }^{2}$ GERAS Centre for Aging Research, ${ }^{3}$ Centre for Family Medicine Family Health Team; School of Pharmacy, University of Waterloo, ${ }^{4}$ Centre for Family Medicine Family Health Team

Background: COVID-19 pandemic restrictions and reduced access to services posed potential risks for frail older adults. The Centre for Family Medicine (CFFM) created a telephone outreach risk assessment protocol to identify potential health and psychosocial risks so that proactive interventions could be initiated to prevent destabilization/crisis. This study describes patient/caregiver and clinician perceptions of this outreach program.

Methods: 335 patients previously identified as frail in the CFFM Case-finding for Complex Chronic Conditions in adults aged $75+(\mathrm{C} 5-75)$ program, or as vulnerable by their physician were screened by nursing staff between April 1 and May 20, 2020. Screening included risk of social isolation, health destabilization, medication related problems, lack of access to services/supports and caregiver stress. Those identified with risks were connected directly to the most appropriate healthcare provider for intervention. Information on COVID-19 prevention was provided. Semi- structured phone interviews were conducted with patients (26), caregivers (5) and clinicians (9) involved in this initiative to better understand their perceptions of the outreach program.

Results: Ten key themes associated with this outreach were identified. For patients/caregivers the outreach: generated a sense of feeling cared for, valued and not forgotten; provided reassurance that healthcare was still accessible; and access to trusted information about the virus. Clinicians valued the outreach as patients were unaware they could access primary care; the protocol was perceived as comprehensive; efficient and feasible to implement; resulted in risk identification and crisis aversion; and was perceived as meaningful work during the pandemic. Both groups supported future implementation.

Discussion: Telephone outreach to patients/caregivers provides a significant opportunity to provide information, support and mitigate risks for frail older adults.

Conclusions: Risk screening is an important component of pandemic planning in primary care.

The association among cognition, frailty, and falls and falls and incident fracture: results from the Canadian Longitudinal Study on Aging (CLSA)

Ahreum Lee ${ }^{1}$, Caitlin McArthur ${ }^{2}$, George Ioannidis ${ }^{3}$, Jonathan D Adachi ${ }^{4}$, Lauren E Griffith ${ }^{4}$, Lehana Thabane ${ }^{4}$, Lora Giangregorio ${ }^{5}$, Suzanne N Morin ${ }^{6}$, William D Leslie ${ }^{7}$, Justin Lee ${ }^{4}$, Alexandra Papaioannou ${ }^{3}$

${ }^{1}$ McMaster University, ${ }^{2}$ Dalhousie University, School of Physiotherapy, ${ }^{3}$ GERAS Centre for Aging Research; McMaster University, Department of Health Research Methods, Evidence, and Impact, ${ }^{4}$ McMaster University, Department of Health Research Methods, Evidence, and Impact, ${ }^{5}$ University of Waterloo, Department of Kinesiology; Schlegel-UW Research Institute on Aging, ${ }^{6}$ McGill University, Department of Medicine, ${ }^{7}$ University of Manitoba, Department of Internal Medicine

Background: The probability of experiencing a fracture in the next 10 years can be calculated using fracture risk assessment instruments like the FRAX. However, current instruments do not include potential risk factors like cognition, frailty, or falls. The purpose of our study is to investigate the association between cognition, frailty, and falls, and fracture risk for community dwelling older adults in the Canadian Longitudinal Study on Aging (CLSA).

Methods: We included participants who completed the 2015 baseline comprehensive interview, did not experience any 
self- reported fracture in the prior year, and completed the 2018 follow-up questionnaire $(\mathrm{n}=26,982)$. We compared the Mental Alternative Test (MAT) for cognition, frailty index (FI) score derived from 42 items, and self-reported falls in the last year for those with and without an incident fracture. We used multivariable logistic regression adjusted for age, sex, ethnicity, educational level, marital status, parental hip fracture history, prior fracture, corticosteroids use, rheumatoid arthritis status, self-reported osteoporosis status, and DXA femoral neck T-score to test for associations.

Results: A total of 739 participants experienced a self-reported incident fracture in the last year at follow-up. Participants who experienced an incident fracture had similar cognition scores [mean (SD) MAT 26.26 (8.77) vs. 26.85 (8.65), $\mathrm{p}=0.0708$ ], higher degree of frailty [mean (SD) FI $0.13(0.08)$ vs. 0.12 $(0.07), \mathrm{p}<.0001]$, and greater percentage had fallen [n (\%) 42 (5.68) vs. 906 (3.45). $\mathrm{p}=0.0023]$. The adjusted odds for experiencing an incident fracture was associated with frailty [adjusted OR 1.17 (95\% CI: 1.02 - 1.35)], but not cognition [adjusted OR $1.00(95 \%$ CI $0.99-1.01)$ ] or falls [adjusted OR $1.41(95 \%$ CI $0.93-2.13)]$.

Discussion: Frailty is associated with incident fracture and could be considered for inclusion in fracture risk assessment instruments.

\section{Conclusions: -}

\section{Ultrasonic Bedside Measures of Muscle Thickness and Sarcopenia in Older Adults}

Kenneth Madden, Boris Feldman, Shane Arishenkoff, Graydon Meneilly

University of British Columbia

Background: The loss of muscle quantity and muscle quality with increasing age is defined as sarcopenia, which is associated with increased morbidity and mortality. Current guidelines to diagnose sarcopenia require the use of Dual Xray Absorptiometry (DXA), a test that cannot be performed at the bedside and is rarely used clinically to assess muscle mass. The increasing use of point-of care ultrasound (PoCUS) is one potential solution. We examined the association between ultrasonic measures of muscle thickness (MT, vastus medialis muscle thickness) and measures of muscle mass and quality in older adults.

Methods: 150 older adults (age $>=65$; mean age $80.0 \pm 0.5$ years, 66 women, 84 men) were recruited sequentially from geriatric medicine clinics. Each subject had lean body mass (LBM, by bioimpedance assay), grip strength, mid-arm biceps circumference (MABC), gait speed, and MT measured. All initial models were adjusted for biological sex.

Results: In our final models, MT showed a strong association with measures of muscle mass, including MABC
(Standardized $\beta=0.141 \pm 0.067, \mathrm{R} 2=0.417, \mathrm{p}=0.038$ ) and LBM (Standardized $\beta=0.204 \pm 0.058, \mathrm{R} 2=0.577, \mathrm{p}<0.001$ ). With respect to muscle quality, there was a strong association with grip strength (Standardized $\beta=0.118 \pm 0.115, \mathrm{R} 2=0.511$, $\mathrm{p}<0.001)$ but no significant association with subject performance (as measured by gait speed).

Discussion: Bedside ultrasound measures of muscle thickness showed strong associations with measures of muscle mass (LBM and MABC) and muscle quality (grip strength).

Conclusions: Although more work needs to be done, bedside ultrasound shows potential as a screening tool for sarcopenia in older adults.

\section{No where to go: Socially vulnerable adults are more likely to have longer hospital stays and be transferred to ALC status awaiting long-term care}

Jasmine Mah ${ }^{1}$, Judith Godin ${ }^{1}$, Susan Stevens ${ }^{2}$, Janice Keefe $^{2}$, Kenneth Rockwood ${ }^{1}$, Melissa Andrew ${ }^{1}$ ${ }^{1}$ Dalhousie University, ${ }^{2}$ Mount Saint Vincent University

Background: Social vulnerability (SV) is the accumulation of poor social circumstances culminating in a person being susceptible to adverse health outcomes. Poor social circumstances have previously been associated with increased mortality, cognitive decline and long-term care (LTC) placement; however, determinants are generally studied in isolation, which no longer reflects the complexity of our ageing population. To address this gap, we examined a holistic measure of SV as a predictor of hospital admission, length of stay (LOS), and discharge destination.

Methods: A retrospective study was conducted using the Geriatric Patient Information Database of older adults presenting to the emergency department. A 12-item Social Vulnerability Index (SVI) and 57-item Frailty Index (FI) were calculated, using a deficit accumulation approach. Unadjusted and adjusted linear and logistic regression models were fitted to estimate associations of the SVI with outcomes obtained from linkage with the Discharge Abstract Database.

Results: In 1,164 patients (mean age 80.5 $\pm 8.3,54.2 \%$ female), mean SVI score was $0.40 \pm 0.16$ and mean FI was $0.44 \pm 0.14$. Amongst patients admitted $(\mathrm{n}=711)$, for every 0.1 unit increase in SVI, LOS increased by 4 days $(\mathrm{p}<0.001)$ after adjusting for age, sex and FI. SVI was associated with staying over the expected LOS (aOR: 6.82, 2.05-22.67, $\mathrm{p}=0.002$ ), becoming alternate level of care (ALC) (aOR 64.16, 7.83-526.06, $\mathrm{p}<0.001$ ) and being discharged to LTC (aOR 41, 10.03-175.37, $\mathrm{p}<0.001)$. SVI was not associated with admission or in-hospital mortality.

Discussion: These findings add to the existing SVI literature, supporting the robustness of a social deficit approach. $\mathrm{SV}$ should be routinely assessed in all patients given the potential for interventions to mitigate outcomes and improve resource efficiency. 
Conclusions: SV, independent of frailty, was associated with increased LOS, becoming ALC, and being discharged to LTC from hospital in multivariate analyses.

\section{Is there interest in a Canadian geriatrics-focused mentorship program between junior medical trainees and subspeciality residents or staff physicians?}

Jasmine Mah, Michael Sun², Josh Goodwin², Bonnie Cheung $^{3}$, Victoria Chuen ${ }^{4}$, Maia von Maltzahn ${ }^{5}$

${ }^{1}$ Dalhousie University, ${ }^{2}$ Department of Medicine, Dalhousie University, Department of Medicine, Dalhousie University, ${ }^{3}$ Department of Medicine, University of Toronto Faculty of Medicine, ${ }^{4}$ Department of Medicine, McMaster University, ${ }^{5}$ Division of Geriatric Medicine, Dalhousie University

Background: Mentorship programs in medicine are proven to benefit trainees and physicians by supporting wellbeing, research productivity, and career preparation. Canadians are ageing and there is a growing need to stimulate interest in specialties for geriatric populations. The Resident Geriatric Interest Group launched a new nation-wide longitudinal mentorship program, which matches junior trainees (medical students or junior residents) with a staff physician or senior trainee in Geriatrics (Internal Medicine), Care of the Elderly (Family Medicine), or Geriatric Psychiatry. We aim to characterize the trainees and mentors who signed up to participate in the program.

Methods: A call for applications was distributed via email and social media between November 15th, 2020 and January 14th, 2021 to all medical schools in Canada. We report findings from the registration questionnaire using descriptive statistics to summarize demographic data, their academic interests and perspectives on mentorship.

Results: Of the 85 mentees, $73.7 \%$ were female, $52.9 \%$ selfidentified as an ethnic minority and $55.8 \%$ were between the ages of $20-25$ years. Medical students comprised $85.9 \%$ of the applicants with representation from all 17 schools. Help with career decisions and development of professional networks were felt to be the greatest benefits of such a mentorship program. A total of 100 mentors were recruited, of which $60.0 \%$ were staff and $40.0 \%$ senior residents. Most staff mentors worked in academic practice $(71.4 \%)$ and specialized in geriatrics $(61.7 \%)$.

Discussion: The number of respondents demonstrates overwhelming interest from both trainees and potential mentors for opportunities and platforms facilitating mentorship; mentees were particularly interested in research and geriatric medicine. This pilot project will continue to collect data on mentee-mentor pairs to evaluate program quality.

Conclusions: There is significant interest across Canada for establishing mentorship relationships in geriatric specialities.

\section{Assessing Nurse and Personal Support Worker Knowledge and Care Practices in Context of Delirium: Development of a Survey Tool}

Tanya Mailhot ${ }^{1}$, Christina Clausen ${ }^{2}$, Laura Crump ${ }^{2}$, MarieEve Leblanc $^{3}$, Lia Sanzone ${ }^{4}$

${ }^{1}$ Université de Montréal, 2 Jewish General Hospital, ${ }^{3}$ Mc Gill University Health Centre, ${ }^{4}$ Mc Gill University

Background: The implementation of delirium prevention, detection and management strategies by nurses and personal support workers (PSWs) according to best practice guidelines contributes to better outcomes for patients. However, little is known about care practices of nurses relative to delirium in acute care settings and no studies have investigated PSW care practices. The study sought to create a standardized survey for nurses and PSWs to evaluate their care practices and knowledge relative to delirium.

Methods: The survey was developed using the theoretical framework outlined by Burns et al. and Eysenback et al. (2008, 2004). To generate the items in the survey a literature review of best practice care guidelines, existing survey tools and barriers and facilitators to care was performed. From this, a group of clinicians and experts in delirium then drafted a survey tool, which was reviewed for clarity from stakeholders. Following this, the survey underwent several rounds of reviews.

Results: The completed nurse survey consists of 38 items answered on a four-point Likert scale, divided into four sections: knowledge, practice, collaboration and confidence. The PAB survey consists of 20 items answered on a four-point Likert scale, divided in four sections: knowledge, care, communication and confidence.

\section{Discussion: N/A.}

Conclusions: This survey tool will be used in an upcoming multi-site study. This tool could be implemented to identify knowledge deficits and barriers and facilitator to optimum delirium care in order to create targeted education programs and other interventions.

\section{The Geriatric Certificate Program: Improving Access to Geriatric Education through a Novel Online Program}

Sharon Marr ${ }^{1}$, Michelle Doherty², Jennifer Siemon ${ }^{2}$, Loretta Hillier ${ }^{3}$

${ }^{1}$ Division of Geriatric Medicine, McMaster University, ${ }^{2}$ Regional Geriatric Program Central, ${ }^{3}$ GERAS Centre for Aging Research

Background: The Geriatric Certificate Program (GCP) provides health care workers with evidence-based geriatric education. Historically, a low proportion of non-regulated workers have registered for the program. Cost and timecommitment were found to be barriers to participation. The 
Online Non-Regulated Stream was developed to address these barriers. This study describes the outcomes and impact of this program as reported by the first group of graduates.

Methods: Upon program completion, all graduates $(\mathrm{N}=21$; $100 \%$ ) completed an online evaluation survey.

Results: Graduates included, personal support workers or health care aides (19\%), students (19\%), physiotherapy assistants $(10 \%)$, educators $(10 \%)$ and recreational therapists $(10 \%)$ among others. Mean ratings (5-point scale: $1=$ poor; $5=$ excellent) show that all courses were well rated (Frailty eLearning: $4.05 \pm 0.97$; U-FIRST! Online: $4.38 \pm 0.92$; eGeriatric Training Program: $3.95 \pm 1.20$; Gentle Persuasive Approaches eLearning: $4.29 \pm 0.72$ ). Most students found the pace $(95 \%)$, level of difficulty $(81 \%)$, and amount of information $(100 \%)$ to be "just right" and $95 \%$ found the courses relevant to their work. For most graduates, the online format $(4.76 \pm 0.44)$, cost $(4.43 \pm 0.81)$ and desire to improve work opportunities $(4.57 \pm 0.68)$ greatly impacted their decision to register. The majority of graduates $(95 \%)$ indicated that they were able to apply information from this program to their work and now feel more confident (85\%) working with older adults. Graduates reported fewer workplace injuries $(28 \%)$ and sick days $(17 \%)$ after the program. All students were either "very much" $(52 \%)$ or "extremely satisfied" (48\%) with the GCP.

Discussion: This program is effective in improving worker outcomes while meeting learning needs.

Conclusions: A specific online program for non-regulated workers can increase their participation in capacity building education.

An updated prevalence-estimate of osteoporosis and fracture risk for community-dwelling older adults: results from the Canadian Longitudinal Study on Aging (CLSA)

Caitlin McArthur ${ }^{1}$, Ahreum Lee ${ }^{2}$, Jonathan D Adachi², Lora Giangregorio ${ }^{3}$, Lauren E Griffith ${ }^{4}$, Suzanne Morin ${ }^{5}$, Lehana Thabane ${ }^{4}$, George Ioannidis ${ }^{2}$, Justin Lee ${ }^{2}$, William D Leslie ${ }^{6}$, Alexandra Papaioannou ${ }^{2}$ ${ }^{1}$ Dalhousie University, ${ }^{2}$ McMaster University, GERAS Centre for Aging Research, ${ }^{3}$ University of Waterloo, Schlegel- UW Research Institute for Aging, ${ }^{4}$ McMaster University, ${ }^{5}$ Mc Gill University, ${ }^{6}$ University of Manitoba

Background: Previous studies have estimated the prevalence of osteoporosis in Canada to be 10.1-15.8\% from administrative data or self-report but were completed 10 to 20 years ago. The purpose of our study is to provide updated prevalence estimates of osteoporosis and fracture risk for community dwelling older adults using the Canadian Longitudinal Study on Aging (CLSA).

Methods: We included participants who completed the baseline (2015) comprehensive interview and had dual-energy X-ray absorptiometry (DXA) $(\mathrm{N}=28,781)$. We describe the age- and sex- stratified prevalence of osteoporosis (Femoral neck T-score $\geq-2.5)$ and high fracture risk (FRAX ${ }^{\circledR}>20 \%$ ) per 1000 persons as well as self-reported lifetime history of major osteoporotic fracture (hip, wrist, spine, pelvis, humerus), and the proportion of participants who self- report taking an osteoporosis medication.

Results: The mean age of participants was 70.0 (standard deviation: 10.3). Overall, $26.6 \%$ of participants had DXAdefined osteoporosis of which $48.6 \%$ reported taking an osteoporosis medication. The prevalence of osteoporosis per 1000 persons increased with age for both men (45-54 years: 188.0 ; $55-64$ years: 206.4 ; $65-74$ years: 246.6 ; $75+$ years: 372.8$)$ and women (45-54 years: 192.9 ; $55-64$ years: 246.3; $65-74$ years: 318.9 ; $75+$ years: 479.4$) .15 .0 \%$ of the participants self-reported a lifetime history of fracture, of which $39.4 \%$ were major osteoporotic fractures. $6.2 \%$ of all participants were at high fracture risk, with the highest prevalence for women over the age of 75 (212.8 per 1000 persons). $14.7 \%$ of people at high fracture risk reported not taking an osteoporosis medication.

Discussion: Our study demonstrates the prevalence of osteoporosis to be higher than previous older studies using administrative data and self-report.

Conclusions: Osteoporosis is highly prevalent among community-dwelling older adults, particularly in older women who have the highest disease and fracture risk prevalence.

\section{The Use of Prisma-7 Questionnaire Within the McGill University Health Centre}

Desmond Milroy ${ }^{1}$, Erica Marrone ${ }^{1}$, Mona Maalouf ${ }^{2}$, José A. Morais ${ }^{1}$

${ }^{1}$ McGill University, ${ }^{2}$ Mcgill University Health Centre

Background: The PRISMA-7 is employed as a frailty screening tool in the McGill University Health Center (MUHC) adult hospitals. This study sought to assess its use in the MUHC and whether a positive PRISMA-7 score $(\geq 4)$ was associated with important hospital use characteristics

Methods: AdatasetwasgeneratedbyqueryingMedUrgeelectronicmedicalrecordintheemergencydepartment(ED)and patient registration databases at the MUHC for the 2017-2018 financial year for patients $\geq 75 \mathrm{y}$ old. Comparisons were made (OR; $\pm 95 \%$ CI) between those who scored $\geq 4$ vs $<4$.

Results: The dataset comprised a total of 12331 ED visits with 8226 unique patients. The PRISMA-7 was completed in $86 \%$ of ED visits and $45 \%$ tested positive (frail) with an increasing frequency with age. $52 \%$ of males tested positive, compared to $38 \%$ of females. In the ED, frail patients stayed longer than $48 \mathrm{~h}(1.43 ; 1.43-1.44)$, had increased geriatrician $(1.94$; $1.92-1.96)$ and specialized nurse consultations $(1.60 ; 1.57-$ $1.63)$, and were more likely to be admitted $(1.69 ; 1.69-1.69)$. Following admission, frail patients had increased likelihood of staying $>1$ week $(1.21 ; 1.20-1.22)$, of placement in long 
term care $(2.00 ; 1.98-2.01)$, and of returning to the ED within 30 days following discharge $(1.53 ; 1.52-1.54)$.

Discussion: WhilethePRISMA-7cut-offof $\geq 4$ isdifferentinthe MUHCcomparedto $\geq 3$ inmanystudies, theincidenceoffrailty is consistent with the literature. More males were identified as frail and there was a peak incidence of frailty after 85 years of age due to specific questions within thePRISMA-7.

Conclusions: The association between a positive PRISMA-7 and important hospital use characteristics is consistent with the PRISMA-7's use as a frailty screening tool within the hospital ED setting.

\section{Developing goals of care for long term care residents during the COVID-19 pandemic}

Paige Moorhouse ${ }^{1}$, Nabha Shetty ${ }^{1}$, Laurie Mallery ${ }^{1}$, Ashley Miller ${ }^{1}$, Maia VonMaltzahn ${ }^{1}$, Anne Marie Krueger Naug ${ }^{1}$, Amanda Tinning ${ }^{2}$, Melissa Buckler ${ }^{1}$

${ }^{1}$ Dalhousie University, ${ }^{2}$ Nova Scotia Health

Background: Background: The COVID-19 pandemic presents a unique circumstance, where care for vulnerable adults in long- term care (LTC) is delivered in a separate location from the most intensive pandemic supports (acute care).

The COVID-19 pandemic presents an opportunity to form relationships to deliver collaborative care. In order to support optimal, appropriate care for LTC residents during COVID-19, internal medicine specialists, and frailty experts began a pilot project ("MED-LTC") to update goals of care for residents.

Methods: Objectives: (1) Identify and understand factors influencing implementation of the program

Explore the enablers and barriers to rapid adaptation of the program acrossNS

Assess the impact of the MED-LTC program on acute care resourceutilization Methods: Mixed Methods Implementation Science

Results: Results: Aspects of exploration to inform program development included need, fit, resources, evidence, adaptability, and sustainability. Qualitative analysis of survey and focus group interview data (from providers and users of the program) using a CFIR construct identified characteristics that supported the innovation; those that affected its implementation, resident and family need related to clinical care and delivery; as well as factors important for further development and roll-out of the Med-LTC program including the need for technology, administrative support, and clinician dedicated time/remuneration.

Discussion: Quantitative analysis indicates a significant impact of the program on the level of care selected by SDMs with a mean 2.3 point change on an ordinal scale of ACP level of intervention.

Conclusions: The Med-LTC program leveraged identified needs during COVID-19 with technology/fee structure opportunities to deliver an innovative and effective clinical program for LTC providers, residents, and their families.

This project was funded through the Nova Scotia COVID-19 Health Research Coalition

\section{Obtaining multidisciplinary healthcare provider feedback on e-learning for driving cessation and dementia}

Gary Naglie ${ }^{1}$, Elaine Stasiulis ${ }^{1}$, Harvir Sandhu ${ }^{1}$, Jahnel Brookes $^{1}$, Brenda Vrkljan², Claudia Mariano ${ }^{3}$, Isabelle Gélinas $^{4}$, Anna Byszewski ${ }^{5}$, Mark J. Rapoport ${ }^{6}$

${ }^{1}$ Baycrest Health Sciences, ${ }^{2}$ McMaster University, ${ }^{3}$ Nurse Practitioners' Association of Ontario, ${ }^{4}$ McGill University, ${ }^{5}$ University of Ottawa, ${ }^{6}$ Sunnybrook Health Sciences Centre

Background: Healthcare providers (HCPs) can play a critical role in facilitating decision-making and supporting people with dementia (PWD) in their transition to non-driving. However, research consistently shows that HCPs lack the knowledge, skills and confidence to effectively support PWD and family carers in the context of driving cessation. As part of our aim to develop an e-learning program to educate multidisciplinary HCPs about driving and dementia we explored HCPs' perspectives on the proposed content and format of our e-learning modules.

Methods: In-depth interviews were conducted with $22 \mathrm{HCPs}$ (physicians, nurse practitioners and occupational therapists) in six Canadian provinces. Participants were asked to provide feedback about their format and content preferences, which included: 1) communicating about driving; 2) addressing the emotional impact; 3 ) assessing driving fitness and reporting; and 4) supporting quality of life post-cessation. Data were examined using a thematic analysis approach.

Results: Participants reported that all content areas should be included. They emphasized the importance of: including early discussions of driving; addressing the emotional impact on HCPs as well as on PWD; directing content to general practitioners who have less experience and skills, but more contact with PWD; and focusing on challenges faced by PWD and HCPs in rural areas. Asynchronous learning with opportunities for live discussions and case study vignettes were preferred because they offered flexibility and a format to learn about realistic and complex issues encountered in practice.

Discussion: As participants' responses indicated, driving cessation for PWD is a complex and challenging issue requiring $\mathrm{HCPs}$ to be informed and proactive.

Conclusions: Obtaining HCPs' perspectives on the development of e-learning modules is critical to ensuring that it will effectively prepare HCPs to support PWD in the driving cessation process. 


\section{Developing the Driving and Dementia Roadmap (DDR): A Knowledge-to-Action Process}

Gary Naglie ${ }^{1}$, Elaine Stasiulis ${ }^{1}$, Sarah Sanford ${ }^{1}$, Michel Bédard $^{2}$, Patricia Belchior ${ }^{3}$, Anna Byszewski ${ }^{4}$, Alexander Crizzle $^{5}$, Isabelle Gélinas ${ }^{3}$, Shawn Marshall ${ }^{4}$, Frank Molnar ${ }^{4}$, Mark Rapoport ${ }^{6}$

${ }^{1}$ Baycrest Health Sciences, ${ }^{2}$ Lakehead University, ${ }^{3}$ Mc Gill University, ${ }^{4}$ University of Ottawa, ${ }^{5}$ University of Saskatchewan, ${ }^{6}$ Sunnybrook Health Sciences Centre

Background: Despite the well-known challenges that driving cessation poses for people with dementia (PWD) and their family caregivers (FC), there are few evidence-based interventions and resources to support them. To address this gap we used a Knowledge-to-Action approach to develop a Driving Cessation Intervention Framework (DCIF) and Driving and Dementia Roadmap (DDR).

Methods: In the Knowledge Creation phase of the KTA we conducted a series of knowledge synthesis activities including systematic reviews and a meta-synthesis. Knowledge inquiry activities involved qualitative studies with PWD, FCs and healthcare/service providers (HCSP). The second part of this phase, comprised a process of collecting and reviewing available tools and resources, curated according to the DCIF. In the Action Cycle phase we adapted the DDR according to feedback from Alzheimer Society staff and an Advisory Committee of PWD and FCs.

Results: KnowledgeCreationactivitiesresultedintheproductionoftheDCIFandtheDDR. TheDCIFidentifiedkeyelements of the decision-making and transition process to non-driving (e.g. awareness, communication, grief and loss), which along with Action Cycle activities informed our development and refinement of the DDR - a web-based collection of curated tools and resources with guided access to specific content and unique portals for each target audience (i.e. PWD, FCs,HCSPs).

Discussion: In using a KTA framework we employed a systematic approach to the creation, application and promotion of driving cessation knowledge. The involvement of knowledge users in an integrated and iterative manner throughout the development process was essential to ensuring the relevance and uptake of the DDR.

Conclusions: The KTA framework played an integral role in developing a web-based resource with the potential to enhance the safety and quality of life for PWD and FCs.

\section{Redesigning Complex Care Delivery for the 65+: An environmental scan of current available resources for telemedicine in older adults}

Sofia Nene, Roxanne Bennett, José A. Morais, Suzanne N. Morin

McGill University Health Center
Background: The COVID-19 pandemic has pushed healthcare professionals to rapidly seek safe and effective ways to remotely connect with patients. Optimal use of telemedicine in older adults remains poorly understood and adaption challenges persist. We aimed to describe currently available digital platforms and barriers to telemedicine use in this population.

Methods: This environmental scan reviews current literature and publicly available websites for tools and guides on the conduct of telemedicine visits applicable to a tertiary care clinical context. Patient- and healthcare worker- reported barriers and facilitators to telemedicine were also identified.

Results: Three provincial and 18 private telemedicine platforms were identified; while they shared similar structures, private platforms offered a wider range of services and increased interactivity (automated booking and reminder systems, multi-way videoconferencing, etc.). Various tools (checklists, guidelines, etc.) for both practitioners and patients have been published by health and accreditation organizations; however, recommendations adapted to older adults with multiple comorbidities are lacking. Salient barriers to telemedicine in older adults pertained to health (reduced neurosensorial, cognitive, and motor capabilities) or were technology-related (low proficiency, mistrust in technology, and limited access). Healthcare workers reported concerns regarding cost-effectiveness, feasibility of technology implementation and overall quality of care.

Discussion: This review, in conjunction with the results of local surveys of patient and healthcare worker reported barriers currently underway, will inform the development of a guide for patients, caregivers and healthcare professionals to support and facilitate the transition to telemedicine.

Conclusions: Implementing telemedicine requires a comprehensive understanding of current practices. Adapting telemedicine to the needs of older adults will help ensure the continuity of high-quality medical care.

\section{Baseline quality of life and needs of older people in harm reduction housing - implications for service planning}

Lara Nixon ${ }^{1}$, Megan Sampson ${ }^{1}$, Fadzai Moreblessing

Punungwe ${ }^{1}$, Martina Kelly ${ }^{1}$

${ }^{1}$ Department of Family Medicine, Cumming School of

Medicine, University of Calgary

Background: Harm reduction (HR) policy prioritizes younger patients. Older people experiencing homelessness and substance use challenges, represent a growing population in emergency, addiction services. Despite this, HR housing targeting the complex medical needs of seniors is poorly understood. To inform service planning, this study assessed self-reported quality of life (QoL) and care needs of older people living in permanent HR accommodation. 
Methods: Setting: 68-bed supportive living facility providing personal and health supports for men and women aged 55+, with ongoing substance use challenges. A 68 -item in-person survey was developed with resident input, includes validated tools exploring self-reported health status, QoL, service use, substance use, demographics and housing history. All residents were eligible to participate. Descriptive statistics are presented.

Results: Thirty-four participants (50\% of residents), mean age 66 years, ( $75 \%$ male). Participants reported mobility issues (22); moderate-extreme pain (21); moderate-extreme depression or anxiety (16). Despite good access to mental health care, only 8 indicated receiving mental health care in the past 6 months; 28 participants smoked, 12 had problematic alcohol use and 5 used drugs other than alcohol. Only 14 were satisfied with how they spent their time and 17 experienced loneliness several times a month or more.

Discussion: Despite active managed alcohol and tobacco programs, residents experienced a range of physical and mental needs, similar to those of many older people. HR for older people needs to integrate care for addiction with good clinical care, with particular attention to mobility, mental health, pain management and social activities.

Conclusions: Engaging older adults in the design of health and social services through purposeful needs assessment can be hoped to improve the fit of services for these largely overlooked people.

\section{Staff experiences of working with older people in congregate supportive living with Harm Reduction - implications for training and practice}

Lara Nixon ${ }^{1}$, Fadzai Moreblessing Punungwe ${ }^{2}$, Kate

Nuique $^{1}$, Megan Sampson ${ }^{1}$, Martina Kelly ${ }^{3}$

${ }^{1}$ Cumming School of Medicine, University of Calgary, Department of Family Medicine, ${ }^{2}$ Cumming School of

Medicine, University of Calgary, Community Health Sciences, ${ }^{3}$ Cumming School of Medicine, University of Calgary

Background: Growing numbers of older people who use substances are excluded from aged-care settings by abstinenceonly drug policy, prompting calls for integration of harm reduction (HR) and geriatric care. Implementation of HR in supportive housing for older people is uncommon in Canada and under-studied. To inform staff training, this study aimed to gain an in-depth understanding of staff experiences working with older people in a congregate setting which embraces harm reduction policy.

Methods: Qualitative interview study of sixteen participants: (6) healthcare professionals (physicians, nurses, social workers) and (10) unregulated staff. Staff were asked about their experiences working with residents, older people with experiences of homelessness and substance use. Data gathering and thematic analysis were iterative, informed by the Job
Demands-Resources model. Member checking was used to examine our findings and the final interpretation was discussed at staff meetings.

Results: Staff identified personal and organisational resources and demands working in this setting. Personal resources includedtraininginharmreduction, previouspersonalorprofe ssionalexperienceworkingwiththispopulationand personal reward when residents acknowledged their work. Personal demands related to the emotional intensity of working with older people with cognitive impairment and substance misuse. Staff appreciated the variety each day offered. Inconsistency in understandings of harm reduction between staff members, variable levels of support enacting harm reduction and few opportunities for formal team communication contributed to tensions between staffgroups.

Discussion: Baseline understanding of HR principles, clear lines of communication, and intentional structures (including physical space) are needed to protect and promote staff wellness.

Conclusions: Staff were highly motivated and derived high personal satisfaction working in harm reduction settings. Providing ongoing supports and regular communication between team members could enhance seamless delivery of HR.

\section{Geriatric Undergraduate Pharmacy Education : Mapping of Skills Laboratories}

Louise Papillon-Ferland ${ }^{1}$, Valérie Castonguay ${ }^{1}$, Cheryl
Sadowski
${ }^{1}$ Faculty of Pharmacy, University of Montreal, ${ }^{2}$ Faculty
of Pharmacy and Pharmaceutical Sciences, University
of Alberta

Background: Older adults provide unique challenges in delivering pharmaceutical care. Skills laboratories are a mandatory component of pharmacy curriculum and therefore they represent an important opportunity to increase student exposure to geriatric cases and related issues. The objective of this project was to describe geriatric cases included in skills laboratories of an undergraduate pharmacy program.

Methods: A retrospective review of all case-based 2018-2019 skills laboratories (years 1-3) from the Pharm D. program of the Faculty of Pharmacy of University of Montreal was conducted. Laboratory sessions of patient cases aged 65 years and older were selected for analysis. Content was extracted for characteristics relating to the patient, health, medications, and context for care.

Results: 108 skills laboratories were reviewed that included a total of 210 cases. Patients aged 65 years and older represented 51 cases ( $24 \%$ of cases), 8 cases ( $3.8 \%$ of all cases) were patients aged 80 years and older. Geriatric syndromes were present in 4 patient cases. Functional status was available for $10 \%$ of cases, and mobility described for $12 \%$. The median number of comorbidities and medications were 4 and 7 
respectively. Ten patient cases included more than 10 medications and none included more than 15 medications. Potentially inappropriate medications were present in 24 of geriatric cases (47\%) but were specifically discussed in 7 cases only.

Discussion: Very old patients and geriatric problems were present in a minority of patient cases included in pharmacy skills laboratories. Cases including older adults were incomplete for characteristics related to geriatric care.

Conclusions: This mapping of skill laboratories from an undergraduate pharmacy program highlights areas of improvement to increase pharmacy students exposure to geriatric cases and related issues.

\section{Evaluation of Education to Teach the Healthcare Workforce to Provide Person-Centered Care for Family Caregivers of Older People}

Jasneet Parmar ${ }^{1}$, Sharon Anderson ${ }^{1}$, Cheryl Pollard ${ }^{2}$, Lesley Charles $^{1}$, Lisa Poole ${ }^{3}$, Myles Leslie ${ }^{4}$, Gwen McGhan ${ }^{4}$, Carolyn Weir ${ }^{5}$, Charlotte Pooler ${ }^{6}$, Jennifer Stickney-Lee ${ }^{7}$, Kimberly Shapkin ${ }^{8}$

${ }^{1}$ Department of Family Medicine, University of Alberta, ${ }^{2}$ Faculty of Nursing, McEwan University, Program

Director, Care of the Elderly, ${ }^{3}$ Brenda Strafford

Foundation's Dementia Friendly Communities Initiative Committee, AGEWELL Older Adult \& Caregiver Advisory

Committee, Dementia Connections Magazine, ${ }^{4}$ University

of Calgary, ${ }^{5}$ Alberta Health Services, ${ }^{6}$ Alberta Health

Services and Faculty of Nursing, University of Alberta,

${ }^{7}$ Alberta Health Services and Sturgeon Community

Hospital, ${ }^{8}$ Faculty of Nursing University of Calgary and Alberta Health Services

Background: Background: We depend on family caregivers [FCGs] to provide over $80 \%$ of the care for people with dementia yet for the most healthcare providers do not meaningfully involve FCGs as partners in care or support FCGs to maintain their own wellbeing. Recent research recommends health providers receive competency-based education to support FCGs throughout the care trajectory.

Purpose: We introduce our Foundational Competency Based Caregiver-Centered Care Education for the health workforce and report on the mixed methods evaluation.

Methods: Methods: The education was designed to meet the basic educational needs of all health providers who interact with FCGs. The learning resources include six competencyaligned educational modules with videos and interactive exercises. It is offered free online (caregivercare.ca).

We evaluated the impacts of the education at two levels of the Kirkpatrick Barr health workforce training evaluation framework: learner's reaction (Level 1) and changes in knowledge/confidence to work with FCGs (Level 2a, 2b).

Results: Results: In the first 2.5 months, 415 healthcare providers completed the education. Learners were satisfied with the quality of education (Mean 4.75/5; $\mathrm{SD}=.5$ ) and the education increased their motivation to learn more about FCGs (Mean 4.75/5; SD .5). Student's paired samples T-test indicated pre-post education changes in knowledge and confidence to work with FCGs were significant [Pre $(\mathrm{M}=37.8, \mathrm{SD}=7.6)$ to post $(\mathrm{M}=47.2, \mathrm{SD}=3.5) \mathrm{t}(125)=-14.39, \mathrm{p}<.0005$ (twotailed)]Qualitativeresultsderivedfromopenresponsesmirroredthequantitativeresults.

Discussion: Discussion: The health workforce has a role to play in maintaining FCG wellbeing and sustaining the family caregiving that reduces health system costs.

Conclusions: Conclusion: Educating health providers is population health approach to addressing inconsistent system of supports for all FCGs throughout the care trajectory.

\section{Maintenance of Autonomy Through exercise Care during Hospitalization (MATCH): A pragmatic pilot study in hospitalized older adults.}

Eva Peyrusqué, Marie-Jeanne Kergoat, Aline Bolduc, Fanny Buckinx, Caroline Law, Nathalie Veillette, Raquel Fonseca, Mylène Aubertin-Leheudre Centre de Recherche de l'Institut Universitaire de Gériatrie de Montréal

Background: Hospitalization leads to iatrogenic declines due to bed rest which increase health care needs (risk of falls, frailty, use of healthcare services). Even if physical activity (PA) is known as potential solution, it is poorly integrated into hospital usual care due to non-pragmatic available intervention.

Objective : Assess the feasibility, acceptability and effects of implementing a pragmatic systematic, prescribed, specific and unsupervised daily adapted PA training (MATCH) in hospitalized older patients.

Methods: Pilot co-creation study. Of 37 eligible patients hospitalized ( $>65 \mathrm{yrs})$ in geriatric unit, 26 consented to participate and were randomized into two groups: MATCH $(n=13)$ or Control $(n=13)$. MATCH participants received one of the five PA programs ( 3 exercises; 3 times/day) according to the mobility score obtained using a decisional tree.

Feasibility was assessed through adherence, prescription and feedback rates and acceptability using system usability \& Likert scales. Physical performance (balance, gait/walking parameters, muscle strength, muscular capacity) was assessed at admission and discharge. Length of stay and rehabilitation care were also recorded.

Results: In each group, $50 \%$ of participants fell during the last year. Intervention adherence was $83.3 \%$. Participants trained in average 2 sessions/day. Participants enjoyed $(100 \%)$ and were satisfied $(80 \%)$ to MATCH tool. MATCH implementation occurred within 3days. Physician feedback was provided in $90 \%$ of cases. Ninety percent of healthcare professionals found MATCH to be adequate. MATCH-fallers sub-group improved more all physical performance whereas 
MATCH-group increased more the sit-to-stand performance than control groups. MATCH seems to reduce the length of stay and rehabilitation treatment during hospitalization.

Discussion: As feasible, acceptable and safe pragmatic intervention, MATCH could be integrated into usual care to counteract iatrogenic decline.

Conclusions: Further multi-sites RCT studies implementing MATCH are needed to confirm our promising results.

\section{Rurality is Associated with Obesity and Increased Body Mass Index Among Canadians: A Cross- Sectional Study - the Canadian Longitudinal Study on Aging}

Samuel Quan ${ }^{1}$, Verena Menec ${ }^{1}$, Megan O'Connell2, Denise Cloutier $^{3}$, Nancy Newall ${ }^{4}$, Robert Tate ${ }^{1}$, Philip St. John ${ }^{1}$

${ }^{1}$ University of Manitoba, ${ }^{2}$ University of Saskatchewan, ${ }^{3}$ University of Victoria, ${ }^{4}$ Brandon University

Background: Obesity is an important public health concern in both urban and rural settings, but large studies of ruralurban differences of obesity are lacking. Canada's diverse geographic, socioeconomic, healthcare infrastructure, and environmental landscape highlights the need to better understand rural-urban health differences. Our study compared body mass index (BMI) and obesity and along a gradient of rurality in Canada.

Methods: A cross-sectional analysis of self-reported BMI and rurality among Canadians aged $45-85$ years was conducted using secondary data from the Canadian Longitudinal Study and Aging (CLSA). CLSA is a national sample representative of community dwelling residents. Rurality was identified in CLSA based on residential postal codes, which we had then divided into 4 categories: urban, peri-urban, mixed, and rural. Logistic regression was used to calculate adjusted odds ratios (aOR) with $95 \%$ confidence intervals $(95 \% \mathrm{CI})$ between obesity (BMI $\geq 30 \mathrm{~kg} / \mathrm{m} 2$ ) and rurality; adjusting for age, sex, province, marital status, number of residents in household, and household income.

Results: 21,126Canadianresidentssurveyedbetween2010and2015wereincludedinourstudy.Alargeportion(26.8\%)of Canadians were obese. Obesity was less prevalent among urban communities $(25.2 \%)$ than rural $(30.3 \%, \mathrm{p}<0.0001)$, periurban $(28.1 \%, \mathrm{p}<0.0001)$, or mixed communities $(28.7 \%$, $\mathrm{p}<0.0001)$. When compared to urban, adjusted odds of obesity were higher in rural $(\mathrm{aOR}=1.09,95 \% \mathrm{CI}=1.00-1.20)$ and peri-urban $(\mathrm{aOR}=1.20,95 \% \mathrm{CI}=1.08-1.35)$ settings. In areas of mixed urban and rural residence, the association was borderline $(\mathrm{aOR}=1.12,95 \% \mathrm{CI}=0.99-1.27)$.

Discussion: Obesity was less prevalent among urban residents, even after adjusting for socioeconomic variables. Further longitudinal studies are required to examine reasons for why rural-urban differences in obesity are present in Canada.
Conclusions: Living in a rural setting is an independent risk factor for obesity.

\section{A Survey of Older Adults on Essential Content and Features of a Mobile Application (app) for the Management of Acute Pain Post-Fracture}

Natasha Qureshi ${ }^{1}$, Maria Djekic-Ivankovic ${ }^{2}$, Michelle Wall ${ }^{2}$, Gregory Berry ${ }^{3}$, Nancy Mayo ${ }^{1}$, Céline Gélinas ${ }^{4}$, Larry Funnell ${ }^{5}$, Suzanne Morin ${ }^{1}$

${ }^{1}$ Department of Medicine, McGill University and Research Institute of the McGill University Health Centre, ${ }^{2}$ Research Institute of the McGill University Health Centre, ${ }^{3}$ Research Institute of the McGill University Health Centre and Division of Orthopedic Surgery, McGill University, ${ }^{4}$ School of Physical and Occupational Therapy, McGill University, ${ }^{5}$ Ingram School of Nursing, McGillUniversity

Background: Acute pain following an osteoporotic fracture often receives suboptimal treatment that leads to poor outcomes. Considering the benefits of mobile applications (apps) in healthcare delivery, we wish to develop a mobile app to enhance older adults' empowerment in self-management of acute pain following a fracture.Our objective was to seek input on essential features and content of a mobile app for this purpose.

Methods: Using electronic mail, we contacted 7000 members of the Canadian Osteoporosis Patient Network (COPN) to answer a questionnaire developed by an interdisciplinary team. It consisted of 6 closed-ended and 3 open-ended questions. Closed-ended questions were analyzed using descriptive statistics, and conventional content analysis was conducted for open-ended questions.

Results: A total of 305 individuals completed the survey of whom, $82 \%$ were over 60 years old, and $75.4 \%$ had sustained a prior fracture. Most participants (99.7\%) indicated that they own at least one electronic device. Fifty-seven percent indicated that they were 'Very Likely' or 'Likely' to use a mobile application to manage pain following a fracture. A total of 1844 suggestions were provided: maintaining mobility $(\mathrm{n}=427)$ and pharmacologic $(\mathrm{n}=238)$ and non-pharmacologic $(n=276)$ pain management strategies were cited as essential content. Audiovisual qualities such as navigation and layout $(n=335)$ were felt to be key features.

Discussion: The results of the survey indicates that the majority of respondents would be at least likely to use a mobile app for the this purpose, and have provided numerous suggestions for functionalities and content for such an app.

Conclusions: This survey highlights issues important to older adults living with osteoporosis when considering acute pain management following a fracture. This will inform the development of a mobile app to support self-management of acute pain. 


\section{Preventing falls in adults with mild to moderate cognitive impairment: A systematic review and meta-analysis}

Megan Racey ${ }^{1}$, Maureen Markle-Reid ${ }^{1}$, Donna FitzpatrickLewis ${ }^{1}$, Muhammad Usman Ali ${ }^{1}$, Hélène Gagné ${ }^{2}$, Susan

Hunter ${ }^{3}$, Jenny Ploeg ${ }^{1}$, Richard Sztramko ${ }^{1}$, Lisa Harrison ${ }^{4}$, Diana Sherifali ${ }^{1}$

${ }^{1}$ McMaster Unversity, ${ }^{2}$ Ontario Neurotrauma Foundation, ${ }^{3}$ Western University, ${ }^{4}$ Hamilton Health Sciences

Background: Cognitive impairment increases an individual's risk of falls due to the role cognition plays in gait control. Despite research on successful fall prevention interventions, fall rates have remained unchanged as $60-80 \%$ of people with dementia fall within a year. Practitioners are requesting evidence for fall prevention best practices to implement with cognitively impaired adults.

Methods: We conducted a systematic review and meta analysis to identify the effectiveness and implementation strategies of fall prevention interventions in improving falls, fear of falling, gait, balance, and functional mobility. We searched 7 databases for fall prevention interventions involving community-dwelling adults $\geq 50$ years with mild to moderate cognitive impairment. Reviewers screened citations, extracted data, and assessed risk of bias and certainty of evidence (GRADE). We assessed statistical and methodological heterogeneity and performed a meta- analysis of studies.

Results: 509 community-dwelling adults (mean age 67.5 to 84.0 years) with mild to moderate cognitive impairment from 12 RCTs were included in this review. 8 studies were exercise interventions, 3 were multifactorial, and 1 provided medication. Overall, fall prevention interventions had medium significant effects on fear of falling (SMD -0.73 [- 1.10, $-0.36]$ ), balance (SMD $0.66[0.19,1.12]$ ), and functional mobility (SMD -0.56 [-0.94, -0.17]) outcomes and small significant effects on gait control (SMD $0.26[0.08,0.43])$ with moderate certainty of evidence.

Discussion: The effect of fall prevention interventions on direct outcomes, such as falls, remains unclear. There is inadequate reporting of implementation elements which makes it difficult to translate these interventions into practice.

Conclusions: Exercise and multifactorial fall prevention interventions are effective at improving outcomes related to falls, however, longer studies with adequate sample sizes are needed to determine their effectiveness of falls directly.

\section{Health care providers' experiences supporting older homeless adults in outreach settings: An interpretive description study}

Veronica Sacco ${ }^{1}$, Paul Stolee ${ }^{1}$, Veronique Boscart ${ }^{2}$, Ellen MacEachen ${ }^{1}$

${ }^{1}$ University of Waterloo, ${ }^{2}$ Conestoga College Institute of Technology and Advanced Learning
Background: Homelessness among older adults is increasing. Health care providers working with this population have a unique understanding of how services for older homeless adults could be improved; however, there is limited information on their experiences. This study's aim was to gain a better understanding of the experiences (roles, challenges and rewards) of health care providers who support older homeless adults (aged 50+) in outreach settings.

Methods: This research was guided by Thorne's (2008) interpretive description approach. Semi-structured interviews were conducted with 10 health care providers who work in outreach settings. Concurrent data collection and analysis, along with an iterative approach, were used to conceptualize key themes in the data.

Results: Four major themes emerged: 1) the client-provider relationship as an essential building block; 2) progression of care that acknowledges the 'whole person'; 3 ) collaboration as integral to providers' work; and 4) system navigation. Across themes, providers highlighted challenges including insufficient resources and supports, cross- sectoral care coordination, the cyclical nature of homelessness, and stigmatization. Providers also discussed the rewarding aspects of their roles, such as witnessing their clients' resilience, celebrating the small wins, having strong interprofessional relationships with colleagues, and work flexibility.

Discussion: This small exploratory study highlighted that these providers' roles extend beyond clinical services. Providers attained personal and professional fulfillment through their work, but were frustrated by systems-level challenges that imposed barriers to delivering optimal care to clients. Findings from this study informed recommendations for practice, policy and future research.

Conclusions: A greater understanding of these health care workers' experiences can be used to identify strategies on how to further support providers in their roles and enhance service provision for older homeless individuals.

\section{The Association Between Orthostatic Hypotension and Cognitive Performance in Those with Established Cognitive Impairment}

Malcolm Sherwood ${ }^{1}$, Jacquie Baker ${ }^{2}$, Shabaz Malik ${ }^{3}$, Micheal Borrie ${ }^{4}$, Jennifer Fogarty ${ }^{4}$, Jaspreet Bhangu ${ }^{4}$ ${ }^{1}$ Schulich School of Medicine and Dentistry, ${ }^{2}$ Cummings School of Medicine, ${ }^{3}$ Kansas City University of Medince and Biosciences, ${ }^{4}$ St. Joseph's Healthcare

Background: Orthostatic hypotension $(\mathrm{OH})$ is defined as a drop in systolic blood pressure of $20 \mathrm{mmHg}$, or diastolic blood pressure of $10 \mathrm{mmHg}$ within 3 minutes of standing or head-up tilt. $\mathrm{OH}$ has been linked with an increased incidence of cognitive impairment (CI) in longitudinal studies, but its effect in those with established cognitive impairment remains unclear. The aim of this study was to investigate the 
association between $\mathrm{OH}$ and cognition in a nationally representative cohort of community dwelling older adults with cognitive impairment.

Methods: A retrospective, cross-sectional study from The Comprehensive Assessment of Neurodegeneration and Dementia Study (COMPASS-ND). Neuropyschological and clinical data from 445 participants (356 cognitively impaired: Mild Cognitive Impairment and Alzheimer's disease, and 89 healthy controls) were collected from 13 centers across Canada. Cognitive function was assessed using standardized neuropsychological cognitive batteries. Correlational analysis between cognition and $\mathrm{OH}$ was performed using Pearson correlation. An ANCOVA was used to investigate for confounding variables. Fisher $Z$ test was performed to assess significance of subgroup correlational analysis.

Results: A Pearson correlation showed participants with $\mathrm{OH}$ and $\mathrm{CI}$ had lower scores on MoCA $(\mathrm{R}=-0.168) \mathrm{DST}$ $(\mathrm{R}=-0.142)$, verbal fluency $(\mathrm{R}=-0.111)$, and the trail making test $(\mathrm{R}=-0.123)$. The correlations for the MoCA, DST remained significant when controlled for age, sex, and education ( $\mathrm{p}=0.011$ and 0.028 , respectively). $\mathrm{OH}$ was not a statistically significant risk factor for presence of CI $(\mathrm{OR}=1.012[.874-1.173])$.

Discussion: These data suggest that CI adults are more susceptible to the cognitive effects of $\mathrm{OH}$. These results urge further research into the pathophysiological effects of $\mathrm{OH}$ in patients with CI.

Conclusions: In this nationally representative cohort, those with $\mathrm{OH}$ and established $\mathrm{CI}$ demonstrated poorer cognitive performance on a number of common neuropsychological tests compared to their $\mathrm{CI}$ cohorts without $\mathrm{OH}$.

\section{Mental Health Impacts of the COVID-19 pandemic among older women and men in Canada}

Shailee Siddhpuria ${ }^{1}$, Christina Reppas-Rindlisbacher ${ }^{2}$, Alyson Mahar ${ }^{3}$, Rachel Savage ${ }^{2}$, Julie Hallet ${ }^{4}$, Paula Rochon ${ }^{2}$

${ }^{1}$ University of British Columbia, ${ }^{2}$ Women's College Research Institute, ${ }^{3}$ University of Manitoba, Community Health Sciences, ${ }^{4}$ University of Toronto, Department of Surgery

Background: The COVID-19 pandemic may have dire consequences to older adults' mental health and may impact older men and women differently. Our aim was to examine the mental health symptoms of Canadian older adults and determine any discrepancies of symptoms between older men and women.

Methods: We administered an online nationwide crosssectional survey between May 1st and June 30th, 2020 to a convenience sample of older adults aged $\geq 55$ years with longitudinal follow-up between October and December, 2020. We measured self-reported pandemic related mental health symptoms using standardized scales for anxiety, depression and loneliness. Multivariable logistic regression was used to compare the odds of depression, anxiety and loneliness between men and women, adjusting for relevant confounders.

Results: There were 1541 respondents (67.8\% women, mean age $69.3 \pm 7.8$ ). $23.3 \%$ of respondents reported symptoms of depression ( $29.4 \%$ women, $17.0 \%$ men), $23.2 \%$ reported symptoms of anxiety ( $26.0 \%$ women, $19.0 \%$ men) and $28.0 \%$ were lonely ( $31.5 \%$ women, $20.9 \%$ men). 807 respondents completed the follow-up survey. After adjustment for confounders, the odds of reporting elevated depressive symptoms were 2.07 times higher in women compared to men (OR 2.07 [95\% CI 1.50-2.87] $\mathrm{P}<0.0001)$ at baseline. The odds of reporting symptoms of anxiety and loneliness were also higher for women. Mental health symptoms did not change significantly in follow- up survey.

Discussion: Self-reported mental health symptoms were commonly reported by older Canadians, especially older women, throughout the first 8 months of the pandemic. Measures did not change significantly during longitudinal follow- up even when the number of COVID-19 cases were increasing.

Conclusions: Older women were more likely to report pandemic related concern and mental health symptoms compared to men, an important mental health need that should be addressed.

\section{A HIP Quality Improvement Initiative - Utilizing Automated Electronic Admission Order Sets to Improve Hip Fracture Care in South Western Ontario}

\author{
Luxey Sirisegaram ${ }^{1}$, Aquila Gopaul ${ }^{2}$, Jenny Thain ${ }^{3}$ \\ ${ }^{1}$ University of Western Ontario, ${ }^{2}$ University of Calgary, \\ ${ }^{3}$ Schulich School of Medicine
}

Background: $70-90 \%$ of fragility hip fractures are due to osteoporosis. In 2013/2014, OOS* reported approximately $80 \%$ of Ontarian hip fracture patients did not receive investigations nor treatment for osteoporosis after 6 months. The Hip Fracture Intervention Program (HIP), launched in 2018, is a multi-intervention program at Victoria Hospital aimed at improving hip fracture care as per HQO** quality standards (QS). This QI initiative focuses on QS 14 - Osteoporosis Management. The AIM is to improve in-patient osteoporosis management (assessment, investigation, treatment, followup) in patients aged $\geq 65$ with fragility hip fracture, to $70 \%$ within one year.

Methods: HIP created a standardized pre-operative powerplan with automatic requisition for geriatric consultation and osteoporosis bloodwork. Geriatricians performed bone health assessment as part of $\mathrm{CGA}^{* * *}$. Inclusion criteria were: age $\geq 65$ years, fragility intertrochanteric or subcapital hip fracture, admission to B9-200. Baseline (BL) and intervention (INT) data were analyzed. Variables were: powerplan usage, osteoporosis bloodwork (BW), bone health assessment, in-patient 
initiation of antiresorptive treatment and discharge bone health follow up plan.

Results: First QI cycle: Intervention initiated in October 2018. 38 baseline patients, age (years) $82.6+/-7.5,72$ intervention patients, aged $82.7+/-8$. Percentage patients with appropriate BW (BL/INT): 25-hydroxyVitamin D (21/68), Calcium (44/72), Albumin (28/56), TSH (18/46), geriatric consults (18/72), vitamin D supplementation (73/96), discharged on antiresorptive therapy (27/31) and discharge follow up plan (39/83).

Discussion: HIP achieved the aim of improving bone health assessments, vitamin D supplementation and discharge follow up plan to over $70 \%$. BW completion was variable. Rates of in-patient anti-resorptive initiation remained low but with high rates of follow-up arranged. Areas for improvement in cycle 2 are interventions to improve BW requests and in-hospital initiation of anti-resorptive therapy.

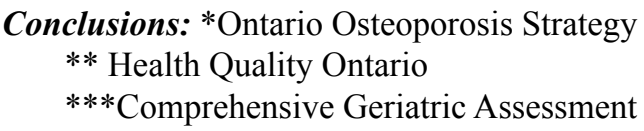

\section{Exploration of Residents' Perception and Responses to the Multiple Dimensions of Clinical Complexity when Caring for Older Adults}

Jenny Thain, Laura Diachun, Lorelei Lingard, Sayra Cristancho

Western University

Background: Managing complex situations is a core feature of physician competency. However, what constitutes 'complexity in clinical medical practice' is not well defined. With implementation of CBME, establishing key features for competency is required. Previous studies involving Attendings and Medical Students demonstrated that complexity extends beyond the medical dimension. Resident perceptions have yet to be explored.

Methods: Constructivist grounded theory was used to inform data collection and analysis. 22 residents PGY-2+ were recruited. Residents were asked to draw Rich pictures, a graphic elicitation technique, based on complex older adults they have cared for. This was used as the basis for discussion and a semi-structured interview. Recorded interview transcripts were analyzed iteratively, identifying themes until data sufficiency was reached.

Results: Two dominant types of complexity emerged medical and social. Their level of challenge over time was paradoxical. Medical complexity was initially perceived as challenging but became progressively easier. Conversely, social complexity became increasingly challenging. Factors associated with this increased challenge included paucity of training in medical school with little exposure during early residency, followed by an abrupt transition in senior residency when residents are expected to competently navigate socially complex issues. This is compounded by a discharge focused hidden curriculum pervasive within hospital culture.

Discussion: Residents are caught in a dichotomy of values between the taught curricula and hospital priorities where patient flow is highly valued. Medical education values medical complexity with little emphasis on social complexity.

Residents, with poor foundational knowledge, are expected to manage socially complex issues to facilitate timely discharges, leading to feelings of frustration, overwhelm and failure.

Conclusions: Social complexity should be considered when designing curricula and key features for determining competence in 'complexity in clinical medical practice'.

\section{The Care-of-the-Elderly Health Guide}

Jean Triscott, Bonnie Dobbs, Lesley Charles, James Huang, David Moores, Peter George Jaminal Tian, Judith Cerna Division of Care of the Elderly, Department of Family Medicine, University of Alberta

Background: The Care-of-the-Elderly Health Guide is a clinical record with cross-references to care recommendations for the elderly. In 2003, the Guide was endorsed by the Health-Care of the Elderly Committee of the College of Family Physicians of Canada. The Guide has been used as a checklist and a monitoring tool for care to older persons. We will update the Guide with current published evidence-based recommendations.

Methods: This study used mixed methods. In Phase 1, the investigators created an initial list of topics and chose published recommendations relevant to family practice. This initial list was reviewed by family physicians in two focus groups. In Phase 2, a panel of ten Care of the Elderly physicians and geriatricians, through a modified Delphi technique with two rounds of online surveys, reviewed whether the recommendations represented current standards of care.

Results: In Phase 1, an initial list of 43 topics and selected recommendations were reviewed by family physicians $(n=12)$ in two focus groups. The focus group discussions had overarching themes of user-friendliness, relevance to clinical practice, and quality of evidence. In Phase 2, in the first online survey, the panel of physicians agreed that 63 of the 66 groups of recommendations (95\%) represented current standards of care. The other 3 groups of recommendations were modified and included in the second online survey, now achieving consensus.

Discussion: The final Health Guide has 47 topics divided into 6 sections. The Guide will be accessible at no cost and be regularly updated.

Conclusions: The Care-of-the-Elderly Health Guide is a quick reference to geriatric care, reviewed for relevance by family physicians and a panel of experts. The Guide will be useful in primary care. 